\title{
Approach to 3-D Ultrasound High Resolution Imaging for Mechanically Moving Large-Aperture Transducer Based upon Fourier Transform
}

\author{
Zalman M. Benenson, Alexey B. Elizarov, Tatiana V. Yakovleva, \\ and William D. O'Brien, Jr., Fellow, IEEE
}

\begin{abstract}
A new three-dimensional (3-D) acoustic image formation technique is proposed that is based on the transmission of wide bandwidth pulse signals and the application of the 3-D fast Fourier transform. A solution to the Helmholz wave equation has been obtained using the Born approximation. The solution contains analytical expressions for the spatial spectra of the transmit and receive radiation patterns for transducers of various geometries with lenses of fixed focal distances. It has been shown that the proposed algorithms allow for radiation patterns with constant widths at depths both behind and in front of the focal point, starting practically from the plane of the transducer. The theoretical and experimental investigations and computer simulation for both spherical and rectangular transducer shapes have been performed. The results were used to estimate the beamwidths and the side lobe levels. A variant of the linear array has been studied for cylindrical lens of a fixed focal distance moving in a lateral direction. It has been shown that, in this case, a high resolution (of the order of a few wavelengths) can be achieved along all three Cartesian coordinates at a very high scanning velocity. The influence of the moving scatterers' velocity in inhomogeneous medium on the spatial radiation pattern characteristics has been estimated.
\end{abstract}

\section{INTRODUCTION}

$\mathrm{V}$ OLUMETRIC 3-D high-resolution acoustic images are of significant practical importance both for medical ultrasound imaging and for nondestructive evaluation. At the present time $3-\mathrm{D}$ imaging is realized by means of two principle techniques. The first technique consists in the scanning the transducer with annular array along azimuth and elevation angles [1]. This method allows for achieving the dynamic focusing in both lateral coordinates only for the receiving radiation pattern. However, the data acquisition time in this case is rather long (of the order of $2-3$ seconds), and the sizes and the weight of the probe are sig-

Manuscript received January 31, 2002; accepted August 5, 2002. This work was partially supported by grant RP2-2104 from the U.S. Civilian Research and Development Foundation and by grant N9901-01171 from the Russian Foundation of Basic Research (RFBR).

Z. M. Benenson, A. B. Elizarov, and T. V. Yakovleva are with the Scientific Council on Cybernetics RAS, Vavilova St., 40, r. 232, Moscow, 117333, Russia (e-mail: benenson@mtu-net.ru).

W. D. O'Brien, Jr., is with the Bioacoustics Research Laboratory, Department of Electrical and Computer Engineering, University of Illinois, Urbana, IL 61801. nificantly bigger than those of traditional medical probes for 2-D imaging. Besides, the area that can be imaged by this method does not exceed in size a rather small volume near the transducer's surface. The second technique is realized by means of linear or convex arrays being mechanically moved along the coordinate perpendicular to the longitudinal axis of the array or by rotation [2]-[4]. A cylindrical lens with a fixed focal distance is installed on the rectangular or cylindrical surface of a transducer. The lateral resolution along the coordinate of motion in zones in front and behind the focus is significantly worse than the resolution along the electronic scanning direction. In order to improve the resolution along the coordinate of motion, it is reasonable to consider the application of a signal processing technique that is similar to the synthetic aperture method [5].

In ophthalmology, dermatology, and possibly mammography, very high lateral and axial resolutions are needed, and they can be achieved only at higher frequencies, that is, $20 \mathrm{MHz}$ to $100 \mathrm{MHz}$. At such frequencies it becomes much more difficult to fabricate a linear or phased array. For achieving high resolution while also increasing the signal-to-noise ratio, a large-size aperture (ca. 50 wavelengths) with a fixed focal distance has been proposed that would then implement the so-called method of virtual source elements [6]. This method is a modification of the synthetic aperture method. In this method, a correspondence is established between the position of a small moving element and a focal region. Focusing is implemented by summing the received signals with appropriate time delays. The delays are determined from the distances between the position of a given point reflector and the center of focal region. The detailed description of this method has been given for a 2-D image [7]. The strength of this method consists in the simplicity of calculations for the case of 2-D images. However, being based upon approximate calculations, this method does not allow for a significant decrease of the side lobe levels. For 3-D imaging, the signal processing algorithm is much more complicated. Besides, papers [6] and [7] do not consider the signal processing for the image zone in front of the transducer's focal point.

This technique also allows achieving the focusing in the field before the focus at all values of the depth. However, the method of focusing on the basis of the signal delays for 
a virtual source is not accurate by its principle, and the error increases if the achievable lateral resolution becomes close to the wavelength.

In contrast to the dynamic focusing algorithms based on time delays [5]-[7], a 2-D imaging method has been considered that is based on the spatial Fourier transform of received signals by the coordinate of moving a small-size (from a half of wavelength to a few wavelengths) receivingtransmitting element [8]. The spatial Fourier transform then is multiplied by a focusing term that depends on the depth and the spatial and the central temporal frequencies. After the inverse spatial Fourier transform is calculated, a 3 -D image is synthesized. This technique does not allow for obtaining an optimally narrow radiation pattern for wideband pulse signals, and is not applicable for transducers with a large aperture. The algorithm proposed herein requires fewer calculations than required for the synthetic aperture technique [5].

Another Fourier transform method is applied to focused signals [9]. 2-D and 3-D images are synthesized for moving rectangular-shaped transducers of any size with a lens of fixed focus. The signals as functions of time and two lateral spatial coordinates are 3-D Fourier transformed. Focusing at all depths is implemented by means of transformation of the temporal and spatial spectra as well by transforming the temporal frequency as a function of spatial frequencies. This technique is applicable for wide bandwidth pulse signals and transducers with a large aperture. The technique allows for obtaining a narrow constant width of the radiation pattern (beamwidth) for a wide range of distances, but considers only the Gaussian apodization case.

The work proposed herein develops the approach used in [9] for the synthesis of 2-D and 3-D images with improved lateral and axial resolution if compared with existing 3-D imaging techniques. An improvement of the proposed approach from a detailed theoretical and experimental investigation is provided for transducers of any size with either a spherical or rectangular-shaped aperture as well as for moving linear arrays. The technique is based on the theoretical estimation of the 2-D Fourier transform of the radiation pattern spatial function for all values of the temporal frequency.

In the contrast to the methods based upon the synthetic aperture principle, the nearly constant lateral resolution at a large interval of depths can be obtained for some cylindrically symmetrical excitation (the boundary conditions) on the transducer's aperture. The lateral resolution in this case is approximately equal to the Rayleigh limit in the focal point of ordinary spherical transmittingreceiving transducer with the focal distance equaled to the depth of an investigated area. The methods based upon the wave equation solution at such conditions are called the nondiffraction beam technique (i.e., the limited diffraction beams). The investigations for ultrasound imaging are described, for instance, in [13], [14]. This technique uses an annular array with various apodization functions. In particular, it may be used for 3-D imaging, similar to that described in [1]. The radiation pattern that can be obtained by means of this technique is characterized by increasing the side lobes and by worse lateral resolution if compared with the results of the present paper. As for the strong features of the method having been presented in [13], [14], they consist in the comparative simplicity of its realization and in the possibility to increase the 3-D imaging frame rate by means of concurrent emitting, receiving, and processing of signals.

The present paper considers the following issues. In Section II, a derivation of the 2-D Fourier transform of the transmit and receive transducer radiation patterns is presented using the Helmholz wave equation solution for an inhomogeneous medium, and the Born approximation. Previous studies [10]-[12] do not provide explicit solutions for Fourier transforms of pattern diagrams.

Section III deals with the principles of the signal processing algorithm based on the application of the 3-D Fourier transform. In order to obtain an analytical estimate of the lateral resolution, both theoretical investigation and computer simulation of the proposed algorithm are applied for Gaussian apodization of the transmit and receive apertures. The calculated 3-D Fourier transform of the signals from the moving transducer is multiplied by a function of spatial and temporal frequencies. Then the modification of a temporal frequency is performed. The application of these operations with implementation of 3D inverse Fourier transformation by the spatial frequencies and by the modified temporal frequency allows getting an image with the lateral resolution that is constant at a large interval of depths and corresponds to the lateral resolution in the focal point of transmitting-receiving transducer.

In Section IV, the multidimensional Fourier transform technique is investigated, both theoretically and experimentally, for spherically shaped and large size transducers with a fixed focal distance. However, the Fourier transform expressions of the radiation pattern cannot be obtained analytically. Thus, these functions are determined computationally. Based on these functions, the algorithm described in Section III yields the radiation patterns for 2$\mathrm{D}$ and 3-D image formation for both monochromatic and wide bandwidth signals. In the 2 -D case, the side lobe levels at a large range of depths in front of and behind the focus are significantly higher than that for the 3-D case. This is explained by the fact that the spatial spectrum amplitude of the radiation pattern depends significantly upon the comparative value of signals' spatial frequencies by two lateral Cartesian coordinates $x$ and $y$. In addition, Section IV presents the signal processing results. The signals were obtained experimentally with a phantom by moving a spherical transducer.

In Section V, the investigations similar to those presented in Section IV are conducted for a transducer that is rectangular in shape. It is shown that, in contrast to the spherical transducer case, the spatial spectrum of the radiation pattern for a rectangular transducer can be presented as a product of functions, each function depending upon only one corresponding spatial frequency. Also, in Section V, a numerical simulation has been done for the 
case of the linear array moving along the $y$-coordinate in order to obtain a 3-D image.

The physical experiment for moving the rectangular transducer was simulated by processing the phantom scattered signals of all the receiving elements of a phased array, the transmitting elements of which subsequently emitted ultrasound pulses. Such a data acquisition technique allows for the investigation of numerous variants of the receivetransmit transducer's parameters.

In Section VI, a theoretical investigation is conducted in order to study the influence of the velocity of moving scatterers on radiation patterns synthesized by the technique proposed in this paper. The scatterers motion can be caused both by the motions of some parts of a tissue and by the blood flow. Numerical estimations are given that characterize the radiation pattern broadening due to a lateral component of the scatterers' velocity and the radiation pattern shift due to the longitudinal component of the scatterers' velocity.

A disadvantage of the technique being proposed in this paper consists in the application of the mechanically moving transducer and in the possible influence of the moving scatterers on the deterioration of the radiation pattern. These drawbacks could be eliminated by using 2-D phased arrays for 3-D imaging. However, in this case the resolution would be worse, the ultrasound device would be more complicated, and the creation of such a 2-D array could be problematic, especially for high frequencies, such as 7-20 MHz. Some ways of the partial overcoming of these problems are based on the linear arrays arranged as a Mills cross or sparse arrays. These issues are considered in $[15]-[17]$.

\section{Basic Dependencies for Scanning Using Cartesian Coordinates}

A scheme for scanning in Cartesian coordinates $(x, y, z)$ is shown in Fig. 1. The transmit-receive transducer is moved along axes $x$ and $y$ in the $z=0$ plane. The transducer emits an ultrasound pulse and receives signals (echoes) scattered from the inhomogeneous medium at various depths $z$. Let us denote a function of pressure at time $t$ by $\hat{P}_{1}\left(x^{\prime}, y^{\prime}, z, t \mid x, y\right)$ at position $\left(x^{\prime}, y^{\prime}, z\right)$ in an inhomogeneous medium that is emitted from the transducer at position $(x, y)$ in the $z=0$ plane. The inhomogeneous medium is assumed to be a weak scatterer. The function $\hat{P}_{1}$ satisfies the following equation [10]:

$$
\nabla^{2} \hat{P}_{1}-\left(\frac{1}{c_{0}^{2}}-\frac{2 \delta c}{c_{0}^{3}}\right) \frac{\partial^{2} \hat{P}_{1}}{\partial t^{2}}=\left(\frac{1}{\rho_{0}} \nabla(\delta \rho)\right) \cdot \nabla \hat{P}_{1}
$$

where $\delta c\left(x^{\prime}, y^{\prime}, z\right)$ and $\delta \rho\left(x^{\prime}, y^{\prime}, z\right)$ are small changes of the sound speed and density in the inhomogeneous medium, and $c_{0}$ and $\rho_{0}$ are constant values of the propagation speed

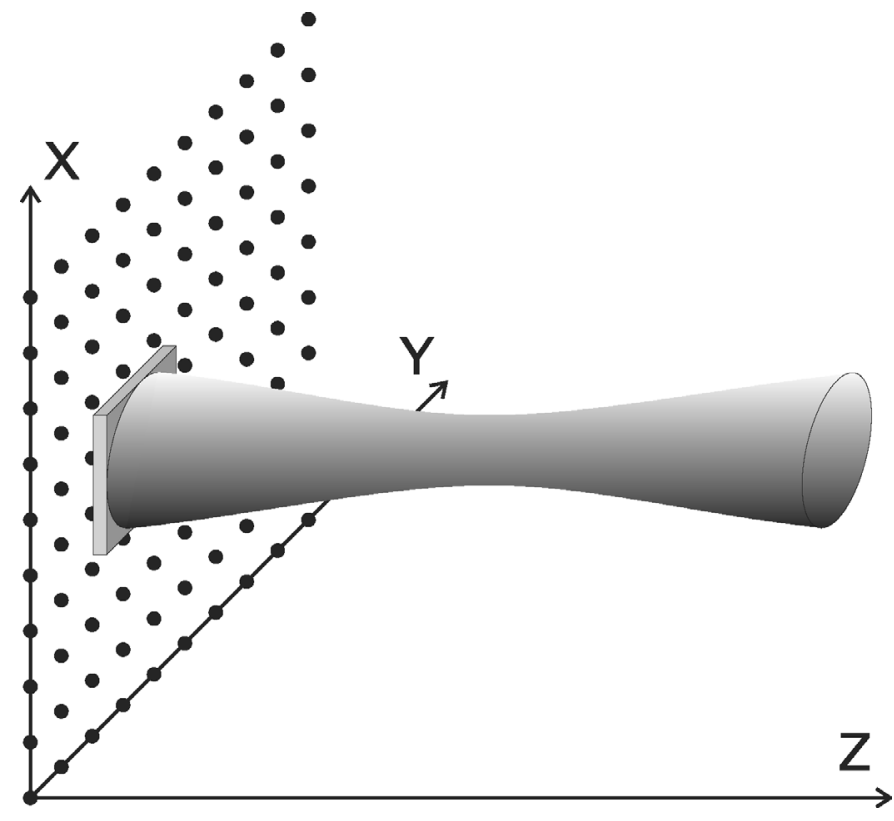

Fig. 1. The scheme of scanning in Cartesian coordinates.

and density. The following notations are used:

$$
\begin{aligned}
& \text { vectors: } \nabla \delta \rho=\left\{\frac{\partial \delta \rho}{\partial x^{\prime}}, \frac{\partial \delta \rho}{\partial y^{\prime}}, \frac{\partial \delta \rho}{\partial z}\right\} \\
& \text { and } \nabla \hat{P}_{1}=\left\{\frac{\partial \hat{P}_{1}}{\partial x^{\prime}}, \frac{\partial \hat{P}_{1}}{\partial y^{\prime}}, \frac{\partial \hat{P}_{1}}{\partial z}\right\} ; \\
& \text { Laplacian operator: } \nabla^{2} \hat{P}_{1}=\frac{\partial^{2} \hat{P}_{1}}{\partial x^{\prime 2}}+\frac{\partial^{2} \hat{P}_{1}}{\partial y^{\prime 2}}+\frac{\partial^{2} \hat{P}_{1}}{\partial z^{2}} .
\end{aligned}
$$

Applying the Born approximation of the first order, we assume that:

$$
\begin{aligned}
& \hat{P}_{1}\left(x^{\prime}, y^{\prime}, z, t \mid x, y\right)= \\
& \hat{P}_{1}\left(x^{\prime}, y^{\prime}, z, t \mid x, y\right)+ \\
& \hat{P}_{s}\left(x^{\prime}, y^{\prime}, z, t \mid x, y\right)
\end{aligned}
$$

where $\hat{P}_{i}$ is the transmitted wave pressure, independent of the inhomogeneities of the medium, where $\hat{P}_{i}\left(x^{\prime}, y^{\prime}, z, t \mid x, y\right)=\hat{P}_{i}\left(x^{\prime}-x, y^{\prime}-y, z, t\right)$ and thus satisfies the uniform wave equation:

$$
\nabla^{2} \hat{P}_{i}-\frac{1}{c_{0}^{2}} \frac{\partial^{2} \hat{P}_{i}}{\partial t^{2}}=0
$$

and $\hat{P}_{s}$ is a pressure that has been scattered by inhomogeneities. It satisfies the following equation:

$$
\begin{aligned}
\nabla^{2} \hat{P}_{s}- & \frac{1}{c_{0}^{2}} \frac{\partial^{2} \hat{P}_{s}}{\partial t^{2}}=-\frac{2 \delta c\left(x^{\prime}, y^{\prime}, z\right)}{c_{0}^{3}} \frac{\partial^{2} \hat{P}_{i}\left(x^{\prime}-x, y^{\prime}-y, z, t\right)}{\partial t^{2}} \\
& +\frac{1}{\rho_{0}} \nabla \delta \rho\left(x^{\prime}, y^{\prime}, z\right) \cdot \nabla \hat{P}_{i}\left(x^{\prime}-x, y^{\prime}-y, z, t\right) .
\end{aligned}
$$

In (6) operators $\nabla$ and $\nabla^{2}$ are applied to coordinates $x^{\prime}$, $y^{\prime}$, and $z$. 


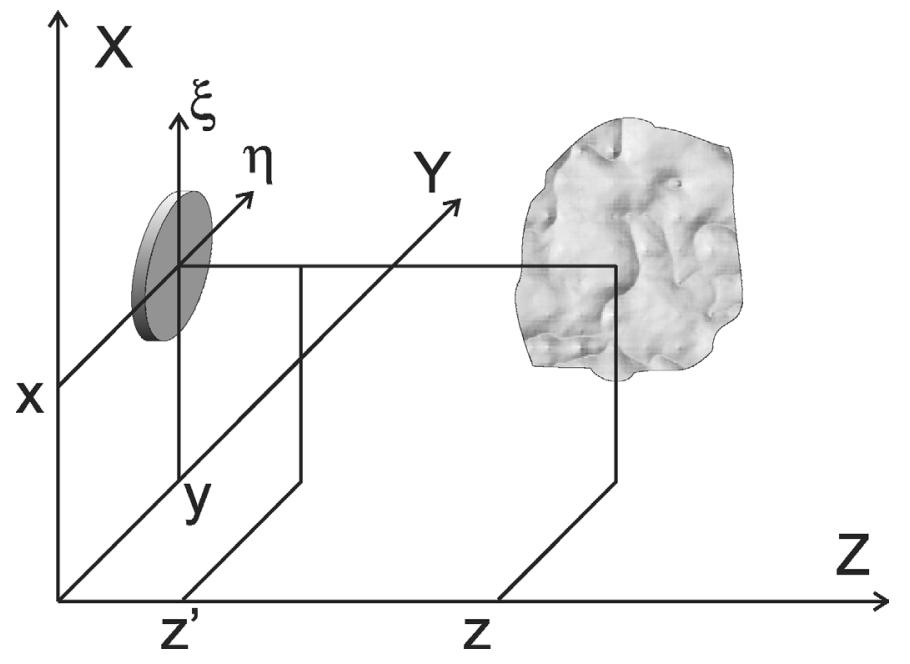

Fig. 2. The scheme of scattering with transducer in a rigid baffle.

Further, let us provide the basic temporal Fourier transform formulas:

$$
\begin{aligned}
P_{i}\left(x^{\prime}-x, y^{\prime}-y, z, \omega\right) & =\int \hat{P}_{i}(\bullet) \exp (-i \omega t) d t, \\
P_{s}\left(x^{\prime}, y^{\prime}, z, \omega \mid x, y\right) & =\int \hat{P}_{s}(\bullet) \exp (-i \omega t) d t,
\end{aligned}
$$

where $\omega$ is the temporal frequency.

The (2) and (3) can be transformed, respectively, to:

$$
\begin{gathered}
\nabla^{2} P_{i}+\frac{\omega^{2}}{c_{0}^{2}} P_{i}=0 \\
\nabla^{2} P_{s}+\frac{\omega^{2}}{c_{0}^{2}} P_{s}=-\frac{2 \delta c\left(x^{\prime}, y^{\prime}, z\right)}{c_{0}^{3}} \frac{\omega^{2}}{c_{0}^{2}} P_{i}\left(x^{\prime}-x, y^{\prime}-y, z, \omega\right) \\
+\frac{1}{\rho_{0}} \nabla \delta \rho\left(x^{\prime}, y^{\prime}, z\right) \cdot \nabla P_{i}\left(x^{\prime}-x, y^{\prime}-y, z, \omega\right) .
\end{gathered}
$$

The source aperture is assumed to be in a rigid baffle. For the transmitted wave, the initial condition in the $z=0$ plane is valid on the active surface $S$ [12] (Fig. 2):

$$
\frac{\partial P_{i}(\xi, \eta, \omega)}{\partial z}=-i \omega f_{1}(\xi, \eta, \omega) \rho_{0} V(\omega),(\xi, \eta) \in S
$$

where $\xi$ and $\eta$ are local coordinates on the active surface. $\frac{\partial P_{i}}{\partial z}=0$ on the $z=0$ surface that is outside of the active surface $S$. The function $V(\omega)$ is the temporal Fourier transform of the vibration velocity of the source aperture that is excited by pulse signals. The complex function $f_{1}(\xi, \eta, \omega)$ is determined by a geometrical shape of the source aperture and/or by its lens. In the function $f_{1}$ the focusing function is taken into account for a given focal distance. We assume that:

$$
P_{i}(x, y, z, \omega)=L_{t r}(x, y, z, \omega) V(\omega) .
$$

The function $L_{t r}(x, y, z, \omega)$ is proportional to the radiation pattern of the transmit transducer at the frequency $\omega$.
Let us denote the Fourier transform of $L_{t r}$ by $x$ and $y$ as:

$$
\begin{aligned}
& u_{t r}\left(\Omega_{x}, \Omega_{y}, \omega, z\right)= \\
& \quad \int \exp \left(-j\left(\Omega_{x} x+\Omega_{y} y\right)\right) L_{t r}(x, y, z, \omega) d x d y,
\end{aligned}
$$

where $\Omega_{x}$ and $\Omega_{y}$ are the spatial frequencies corresponding to the coordinates $x$ and $y$. The Fourier transform of (8) yields an ordinary differential equation for $u_{t r}$ :

$$
\frac{\partial^{2} u_{t r}}{\partial z^{2}}+\left(\frac{\omega^{2}}{c_{0}^{2}}-\Omega_{x}^{2}-\Omega_{y}^{2}\right) u_{t r}=0 .
$$

The solution of this equation for the transmitted wave is:

$$
\begin{aligned}
& u_{t r}\left(\Omega_{x}, \Omega_{y}, \omega, z\right)= \\
& A\left(\Omega_{x}, \Omega_{y}, \omega\right) \exp \left(i z \sqrt{\frac{\omega^{2}}{c_{0}^{2}}-\Omega_{x}^{2}-\Omega_{y}^{2}}\right),
\end{aligned}
$$

where $A$ is some initial condition. Calculating the Fourier transform by $x$ and $y$ from (10) and (11) and differentiating (12) and (14) by $z$, we obtain the final expression for $u_{t r}$ :

$$
\begin{aligned}
& u_{t r}\left(\Omega_{x}, \Omega_{y}, \omega, z\right)=\rho_{0} c_{0} \\
& {\left[\frac{1}{\sqrt{1-\frac{\Omega_{x}^{2}+\Omega_{y}^{2}}{\left(\omega / c_{0}\right)^{2}}}} \exp \left(i z \sqrt{\frac{\omega^{2}}{c_{0}^{2}}-\Omega_{x}^{2}-\Omega_{y}^{2}}\right)\right]} \\
& u_{0}\left(\Omega_{x}, \Omega_{y}, \omega\right),
\end{aligned}
$$

where $u_{0}$ is a Fourier transform of the function $f_{1}$ by $\xi$ and $\eta$ :

$$
u_{0}\left(\Omega_{x}, \Omega_{y}, \omega\right)=\iint f_{1}(\xi, \eta, \omega) \exp \left(-i\left(\Omega_{x} \xi+\Omega_{y} \eta\right)\right) d \xi d \eta .
$$

The term in square brackets in (15) is a Fourier transform of the free-space spatial impulse response for the transmit aperture mounted in a rigid baffle.

We will now determine an expression for the receive radiation pattern. Applying Green's function $G(r)=$ $\frac{\exp \left(i \omega r / c_{0}\right)}{r}, r=\sqrt{\left(x^{\prime}-x\right)^{2}+\left(y^{\prime}-y\right)^{2}+\left(z^{\prime}-z\right)^{2}}$, to solve (9), we obtain an expression for the pressure of the scattered wave $P_{s}\left(\xi, \eta, z^{\prime}-z, \omega \mid x, y\right)$ at the distance $z^{\prime}$ from the point $(\xi, \eta)$ in the receive transducer's plane (Fig. 2):

$$
\begin{gathered}
P_{s}=V(\omega) \iint G(r)\left[\frac{\omega^{2}}{c_{0}^{2}} \frac{2 \delta c\left(x^{\prime}, y^{\prime}, z\right)}{c_{0}} L_{t r}\left(x^{\prime}-x, y^{\prime}-y, z\right)\right. \\
\left.+\frac{1}{\rho_{0}} \nabla \delta \rho\left(x^{\prime}, y^{\prime}, z\right) \cdot \nabla L_{t r}\left(x^{\prime}-x, y^{\prime}-y, z\right)\right] d x^{\prime} d y^{\prime} .
\end{gathered}
$$

An electric signal at the output of the receive transducer can be presented in a form similarly to (10):

$$
\begin{gathered}
V_{r e}(x, y, z, \omega)=\frac{i}{\omega} \frac{1}{\rho_{0}} \iint_{S} \frac{\left.\partial P_{s}\left(\xi, \eta, z^{\prime}-z, \omega \mid x, y\right)\right|_{z=0}}{\partial z^{\prime}} \\
f_{1}(\xi, \eta, \omega) d \xi d \eta f_{2}(\omega),
\end{gathered}
$$


where $f_{2}(\omega)$ is a Fourier transform of the impulse response function of the receive transducer. Substituting (17) into (18) we obtain:

$$
\begin{gathered}
V_{r e}(x, y, z, \omega)=\frac{V(\omega) f_{2}(\omega)}{\rho_{0}} \iint L_{r e}\left(x^{\prime}-x, y^{\prime}-y, z, \omega\right) \\
\times\left[\frac{\omega^{2}}{c_{0}^{2}} \frac{2 \delta c\left(x^{\prime}, y^{\prime}, z\right)}{c_{0}} L_{t r}\left(x^{\prime}-x, y^{\prime}-y, z\right)\right. \\
\left.+\frac{1}{\rho_{0}} \nabla \delta \rho\left(x^{\prime}, y^{\prime}, z\right) \cdot \nabla L_{t r}\left(x^{\prime}-x, y^{\prime}-y, z\right)\right] d x^{\prime} d y^{\prime},
\end{gathered}
$$

where the receive radiation pattern $L_{r e}$ is determined by:

$$
\begin{aligned}
L_{r e}(x, y, z, \omega) & =\left.\frac{1}{\omega} \iint_{S}\left(\frac{\partial}{\partial z^{\prime}} G(\hat{r})\right)\right|_{z=0} f_{1}(\xi, \eta) d \xi d \eta, \\
\hat{r} & =\sqrt{(x-\xi)^{2}+(y-\eta)^{2}+\left(z-z^{\prime}\right)^{2}} .
\end{aligned}
$$

Simple transformations are performed to obtain the Fourier transform $u_{r e}$ of the function $L_{r e}$ by $x$ and $y$ :

$$
\begin{aligned}
& u_{r e}\left(\Omega_{x}, \Omega_{y}, z, \omega\right)= \\
& \quad \iint \exp \left(-i\left(\Omega_{x} x+\Omega_{y} y\right)\right) L_{r e}(x, y, z, \omega) \\
& \quad=\exp \left(i z \sqrt{\frac{\omega^{2}}{c_{0}^{2}}-\Omega_{x}^{2}-\Omega_{y}^{2}}\right) u_{0}\left(\Omega_{x}, \Omega_{y}, \omega\right) .
\end{aligned}
$$

The first factor in (21) is the Fourier transform of the free-space spatial impulse response for the receive aperture mounted in a rigid baffle. Comparing (15) and (21), one can notice that the free-space spatial impulse responses are different for the transmit and receive transducers mounted in a rigid baffle. This is caused by the difference in Green functions for the wave equation solution with the initial conditions that correspond to the case of the transmit transducer, and for the nonuniform wave equation solution that corresponds to the case of the receive transducer. The larger the maximum values of $\left|\Omega_{x}\right|$ and $\left|\Omega_{y}\right|$, the more different are the functions of the receive and transmit radiation patterns. It is noticeable that $\left|\Omega_{x}\right|<\frac{\omega}{c}$ and $\left|\Omega_{y}\right|<\frac{\omega}{c}$ for the waves propagating in space. Besides, it is interesting to note that this free-space spatial impulse response for receiving coincides with that for light irradiated from a large aperture [18].

Formula (19) in square brackets contains two components. The first one corresponds to the so-called monopole scattering that is determined by the variations of the medium's sound velocity $\delta c$. The second component corresponds to the dipole scattering determined by the medium's density variations $\delta \rho$.

Let us consider two characteristic cases of the behavior of the dipole scattering component. Assume that $\delta \rho$ is a smooth function. The first derivatives by $x^{\prime}, y^{\prime}, z$ are significantly less than the ratio $\omega / c$ (or $2 \pi / \lambda$, where $\lambda$ is a wavelength). As the monopole scattering component contains a term $(\omega / c)^{2}$, and $\left|\nabla L_{t r}\right|<\omega / c$, the dipole scattering component does not have a significant influence upon the value of the received signal. Assume that $\delta \rho$ is the sum of a smooth function and some isotropic 3-D pulse function with the width on the order of a diffraction-limited resolution. In this case $\nabla \delta \hat{\rho} \cdot \nabla L_{t r}$ is approximately expressed by:

$$
\nabla(\delta \hat{\rho}) \cdot \nabla L_{t r}=\delta \hat{\rho}_{m} \nabla^{2} L_{t r}
$$

where $\delta \hat{\rho}_{m}$ is a mean value of the 3 -D pulse.

The function $L_{t r}$, as well as $P_{i}$, satisfies (8) and, consequently, $\nabla^{2} L_{t r}=-\frac{\omega^{2}}{c_{0}^{2}} L_{t r}$. In this case, the influence of (22) on the output value of the receive transducer voltage has the same form as the component caused by the monopole scattering. Thus, lateral resolution will be determined by the behavior of the product of the transmit and receive radiation patterns $L_{t r}(x, y, z, \omega)$ and $L_{r e}(x, y, z, \omega)$. The expressions deduced in this section will be used for modeling various radiation patterns and for constructing the signal processing algorithms for variants of transducer shapes and scanning techniques.

It follows from (18) that the axial resolution is determined by the frequency bandwidth of the Fourier transform function $V(\omega)$ of the transducer excitation pulse, by the frequency bandwidth of the impulse response function $f_{2}(\omega)$ of the receive transducer and, in general, by $f_{1}(\xi, \eta, \omega)$.

\section{Development of the Signal Processing Algorithm}

A. Gaussian Apodization Function and an Approach to the Algorithm Construction

Consider the aperture function:

$$
f_{1}(\xi, \eta, \omega)=\exp \left(-\frac{\xi^{2}+\eta^{2}}{a^{2}}\right) \exp \left(-\frac{i \omega}{c_{0}} \frac{\xi^{2}+\eta^{2}}{2 F}\right)
$$

The first term corresponds to the Gaussian apodization function, and the value $2 a$ can be approximately chosen equal to the size of transmit aperture. The second term determines the focusing lens function with the focal distance $F$. The Fourier transform of this function by the coordinates $\xi$ and $\eta$ has the form:

$$
u_{0}^{(h)}\left(\Omega_{x}, \Omega_{y}, \omega\right)=K_{1} \exp \left(-\left(\alpha+i \zeta_{0}\right)\left(\Omega_{x}^{2}+\omega_{y}^{2}\right)\right),
$$

where $\alpha=\frac{1}{4\left(\frac{1}{a^{2}}+\frac{\omega^{2}}{c_{0}^{2}} \frac{a^{2}}{4 F^{2}}\right)}, \zeta_{0}=-\frac{a^{2} \omega / 2 c_{0} F}{4\left(\frac{1}{a^{2}}+\frac{\omega^{2} 2}{c_{0}^{2}} \frac{a^{2}}{4 F^{2}}\right)}$, and $K_{1}$ is a scale coefficient.

Substituting (24) into (15) and (21), and limiting ourselves by the approximation $\sqrt{\frac{\omega^{2}}{c_{0}^{2}}-\Omega_{x}^{2}-\Omega_{y}^{2}} \approx \frac{\omega}{c_{0}}-\frac{\Omega_{x}^{2}+\Omega_{y}^{2}}{2 \omega / c_{0}}$ that is valid in practice for medical ultrasound imaging and nondestructive evaluations at $\left|\Omega_{x}\right|,\left|\Omega_{y}\right|<0.6 \frac{\omega}{c_{0}}$, we 
obtain the Fourier transforms of transmit and receive radiation patterns:

$$
\begin{gathered}
u_{t r}^{(h)}\left(\Omega_{x}, \Omega_{y}, \omega, z\right) K_{2} \frac{1}{1-\frac{1}{2} \frac{\Omega_{x}^{2}+\Omega_{y}^{2}}{\left(\omega / c_{0}\right)^{2}}} \\
\exp \left(-(\alpha+i \zeta)\left(\Omega_{x}^{2}+\Omega_{y}^{2}\right)\right) \exp \left(i z \frac{\omega}{c_{0}}\right), \\
u_{r e}^{(h)}\left(\Omega_{x}, \Omega_{y}, \omega, z\right) \\
=K_{3} \exp \left(-(\alpha+i \zeta)\left(\Omega_{x}^{2}+\Omega_{y}^{2}\right)\right) \exp \left(i z \frac{\omega}{c_{0}}\right),
\end{gathered}
$$

where

$$
\zeta=\frac{c_{0}}{2 \omega} z+\zeta_{0} \approx \frac{c_{0}}{2 \omega}(z-F)
$$

and $K_{2}, K_{3}$ are scale coefficients.

Performing the Fourier transform of (19) by $x$ and $y$, and assuming that the transducer scanning trajectory sizes along axes $x$ and $y$ are large enough (about $\left.\max _{z}\left(\left|\frac{z-F}{F} 2 a\right|+\frac{\lambda z}{2 a}\right)\right)$ and considering only the modified component of the monopole scattering, we obtain:

$$
\begin{aligned}
\tilde{V}_{r e}\left(\Omega_{x}, \Omega_{y}, \omega, z\right)= & K_{4} V(\omega) f_{2} 2(\omega) \frac{\omega^{2}}{c_{0}^{2}} \\
& u\left(\Omega_{x}, \Omega_{y}, \omega, z\right) \Gamma\left(\Omega_{x}, \Omega_{y}, z\right),
\end{aligned}
$$

where $K_{4}$ is a scale coefficient,

$$
\begin{aligned}
& u\left(\Omega_{x}, \Omega_{y}, \omega, z\right)=\iint u_{r e}\left(\Omega_{x}^{\prime}, \Omega_{y}^{\prime}, \omega, z\right) \\
& u_{t r}\left(\Omega_{x}-\Omega_{x}^{\prime}, \Omega_{y}-\Omega_{y}^{\prime}, \omega, z\right) d \Omega_{x}^{\prime} d \Omega_{y}^{\prime}
\end{aligned}
$$

is the Fourier transform of the product of radiation pattern functions $L_{t r}$ and $L_{r e}$, and

$\Gamma\left(\Omega_{x}, \Omega_{y}, z\right)=\iint \exp \left(-i\left(\Omega_{x} x^{\prime}+\Omega_{y} y^{\prime}\right)\right) \gamma\left(x^{\prime}, y^{\prime}, z\right) d x^{\prime} d y^{\prime}$

is the Fourier transform $x^{\prime}$ and $y^{\prime}$ of the medium's scattering function $\gamma$ :

$$
\gamma\left(x^{\prime}, y^{\prime}, z\right)=\frac{2 \delta c\left(x^{\prime}, y^{\prime}, z\right)}{c_{0}}+\frac{\delta \hat{\rho}\left(x^{\prime}, y^{\prime}, z\right)_{m}}{\rho_{0}} .
$$

Formula (27) is valid for any radiation pattern function. Substituting (25) into (28) and assuming that $u_{t r}^{(h)} \approx u_{r e}^{(h)}$, which is acceptable under the condition $a \gg \frac{1}{2} \frac{c_{0}^{2}}{\omega^{2}}$ (normally valid in practice), we obtain for the case of Gaussian apodization:

$$
\begin{aligned}
& u^{h}\left(\Omega_{x}, \Omega_{y}, \omega, z\right)= \\
& \quad K_{5} \exp \left(-\frac{\alpha+i \zeta}{2}\left(\Omega_{x}^{2}+\Omega_{y}^{2}\right)\right) \exp \left(2 i z \frac{\omega}{c_{0}}\right),
\end{aligned}
$$

where $K_{5}$ is a scale coefficient.

The expression for $\zeta(26)$ is known a priori. From (31), it then follows that the algorithm development for the received signal processing assures a constant lateral resolution at all depths $z$. Expression (31) is multiplied by $\exp \left(i \frac{\zeta}{2}\left(\Omega_{x}^{2}+\Omega_{y}^{2}\right)\right)$. In this case the Fourier transform of the resulting radiation pattern will be expressed by the term $\exp \left(-\frac{\alpha}{2}\left(\Omega_{x}^{2}+\Omega_{y}^{2}\right)\right)$. The lateral resolution $\delta l$ will be proportional to $\sqrt{\alpha / 2}$, and, as it follows from (24), it will not depend upon the depth. For the $-6 \mathrm{~dB}$ level, $\delta l \approx \frac{\sqrt{8} F}{a \omega / c_{0}} \approx \frac{F \lambda}{2 a}$ (it is assumed that the aperture is large enough: $\left.\frac{1}{a^{4}}<\frac{\omega^{2}}{4 c_{0}^{2}} \frac{1}{F^{2}}\right)$. This lateral resolution occurs both in the zones behind and in front of the focus.

\section{B. The Principles of the Signal Processing Algorithm Construction}

Following (27) and the other expressions obtained for Gaussian apodization function, we propose the stages of the signal processing algorithm:

- Form the 3-D array of received signals for the discrete values in Cartesian coordinates of the transducer's positions $(x, y)$ in the $z=0$ plane as a function of time $t$.

- Implement the fast discrete Fourier transform by coordinate $t$, and write the values obtained for $\omega>0$ into memory.

- Implement the fast discrete Fourier transform by coordinates $x$ and $y$.

- Multiply the obtained values of the 3-D array as a function of variables $\left(\omega, \Omega_{x}, \Omega_{y}\right)$ by the function $\exp \left(i \zeta\left(\Omega_{x}^{2}+\Omega_{y}^{2}\right)\right)$ for the given values of $z$.

- Implement the inverse discrete Fourier transform of the data array obtained in a previous step by frequency $\omega$ for the given values of $z$.

- Implement the inverse discrete Fourier transform of the result of step five by variables $\Omega_{x}$ and $\Omega_{y}$.

- Calculate the magnitude of analytical spatialtemporal signal obtained as a result of the inverse Fourier transform for 3-D image formation.

It is easy to see that at implementation of steps four and five the most considerable portion of the calculations is to be done. This is caused by the fact that in step four the calculations for all values of $z$ should be performed, but in step five the inverse discrete Fourier transforms should be calculated for all given values of $z$ at $t=\frac{2 z}{c}$.

In order to decrease the time necessary for the calculations, use the fact that $\zeta(26)$ is linearly dependent upon $z$, and replace step four by calculating a new variable:

$$
\tilde{\omega}=\omega+\frac{c_{0}^{2}}{8 \omega}\left(\Omega_{x}^{2}+\Omega_{y}^{2}\right),
$$

interpolate the 3-D data array of arguments $\left(\Omega_{x}, \Omega_{y}, \omega\right)$ from step three to a new regular grid in coordinates $\left(\Omega_{x}, \Omega_{y}, \tilde{\omega}\right)$, and multiply this array by the factor $\exp \left(i \zeta_{0} \omega(\tilde{\omega})\right)$ [where the function $\omega(\tilde{\omega})$ follows from (32)]. The resulting array is given by:

$$
\begin{array}{r}
\tilde{V}^{(h)}\left(\Omega_{x}, \Omega_{y}, \tilde{\omega}\right)=K_{5} \frac{\omega^{2}(\tilde{\omega})}{c_{0}^{2}} V(\omega(\tilde{\omega})) \\
f_{2}(\omega(\tilde{\omega})) \exp \left(i 2 z \frac{\tilde{\omega}}{c_{0}}\right) \Gamma\left(\Omega_{x}, \Omega_{y}, z\right) .
\end{array}
$$


Now in step five we can implement the fast inverse Fourier transform of the function $\tilde{V}^{(h)}$ by $\tilde{\omega}$.

\section{Mathematical Simulation for the \\ Case of Gaussian Apodization Function}

The algorithm above was tested by computer simulation on a personal computer. The monopole reflectors with reflection coefficient $\gamma$ were distributed at various depths in a medium. The results are provided for the case of point reflectors placed at depths of $0.3 \mathrm{~cm}$ and $6 \mathrm{~cm}$ (Fig. 3). The pulse signal with center frequency $f=7.5 \mathrm{MHz}$ and Gaussian spectrum with bandwidth $\delta f=5 \mathrm{MHz}$ was transmitted. The Gaussian apodization function was cut at the level of $-8 \mathrm{~dB}$ at the aperture edges. The received signal is calculated by (15), (21), (27) and by inverse 3-D Fourier transform of $\tilde{V}_{r e}$ from (27) by $\Omega_{x}, \Omega_{y}, \omega$.

The size of the transducer aperture was $2 a=1.2 \mathrm{~cm}$ and the focal distance was $F=1.8 \mathrm{~cm}$. The Gaussian apodization function had a sharp drop of $-8 \mathrm{~dB}$ to zero at the aperture edges. We performed a simulation of the transducer scanning along both coordinates $x$ and $y$ in a $6 \times 6 \mathrm{~cm}$ square. The received signal is calculated by (17) and (18), and only for monopole scattering, i.e., the simulation has been implemented using the Green function at the emit of the transmitting transducer's signal in a rigid baffle; the received signal is calculated in Born approximation using the Green function for the nonuniform wave equation (1) with boundary conditions for transducer in a rigid baffle. Such an approach to numerical simulation is the most accurate and is developed in Born approximation in [12]. The signal processing was performed with the algorithm described above. Fig. 3 illustrates the results of the simulation as the image cross sections in the XY plane for two values of depth $z$ of the point reflectors. For each of the given depths, a few cross sections are shown with a step $\lambda / 2=0.01 \mathrm{~cm}$ in order to show reflectors' images broadening with depth. This broadening would look like arcs on a 2-D cross-section image (in XZ and YZ planes), typical for a nonfocused signal, and it yields circles in the XY-plane sections. From Fig. 3, before processing, the reflectors' images are significantly broadened. After applying the signal processing algorithm, the size of the point reflectors approximately corresponds to the theoretical estimation of lateral resolution $\delta l=0.03 \mathrm{~cm}$ at the level $-6 \mathrm{~dB}$. The artifacts on Fig. 3 after signal processing are determined by side lobe levels of $-30 \mathrm{~dB}$. The axial resolution is about $0.014 \mathrm{~cm}$. Fig. 4 shows the cross sections of 3-D images in XZ and YZ planes before and after the signals processing for the point reflectors placed in the same way as in Fig. 3. Fig. 4 also illustrates the graphic of the signals amplitudes after their processing. From these graphics one can see that the side lobes level is about $-30 \mathrm{~dB}$ at the depth $z=0.03 \mathrm{~cm}$ and it is $-35 \mathrm{~dB}$ at the depth $z=6.0 \mathrm{~cm}$. Such a side lobes level can be explained by cutting the Gaussian apodization function and by adopted approximation in the algorithm.

\section{Investigation of a Spherical Transducer}

\section{A. Theory}

Consider a transducer with a spherically shaped surface that is commonly used in nondestructive evaluation. The transducer radius is $a$, and the radius of curvature is equal to focal distance $F$. Assume that the transducer surface is excited in such a way that all its points vibrate with the same vibrating velocity along the $z$-axis. Also, in the $z=0$ plane, the vibrating velocity at frequency $\omega$ can be expressed as (see also [12]):

$$
V_{1}(\xi, \eta, \omega)=-i \omega \exp \left(-i \frac{\omega}{c_{0}} \frac{\left(\xi^{2}+\eta^{2}\right)}{2 F}\right) V(\omega)
$$

where $V(\omega)$ is a Fourier transform of the excitation pulse. The Fourier transform of the exponential term in (34) by $\xi$ and $\eta$ is:

$$
\begin{aligned}
& u_{0}^{(s)}\left(\Omega_{x}, \Omega_{y}, \omega\right)= \\
& \iint_{S} \exp \left(-i\left(\Omega_{x} \xi+\Omega_{y} \eta\right)\right) \exp \left(-\frac{i \omega}{c_{0}} \frac{\left(\xi^{2}+\eta^{2}\right)}{2 F}\right) d \xi d \eta \\
& \quad=\int_{0}^{a} r d r \exp \left(-\frac{i \omega}{c_{0}} \frac{r^{2}}{2 F}\right) J_{0}\left(r \sqrt{\Omega_{x}^{2}+\Omega_{y}^{2}}\right),
\end{aligned}
$$

where $S$ is a circle (solid disk) surface, $r=\sqrt{\xi^{2}+\eta^{2}}, a$ is the radius of the circle, and $J_{0}$ is a Bessel function of 0 -th order.

Assuming that $a$ is larger than 20 wavelengths, $\lambda=$ $\frac{2 \pi c_{0}}{\omega}$, we get approximately:

$$
\begin{aligned}
& u_{0}^{(s)}\left(\Omega_{x}, \Omega_{y}, \omega\right)= \\
& A_{0}^{(s)}\left(\Omega_{x}, \Omega_{y}, \omega\right) \exp \left(-\frac{i\left(\Omega_{x}^{2}+\Omega_{y}^{2}\right) F}{2 \omega / c_{0}}\right),
\end{aligned}
$$

where $A_{0}^{(s)}\left(\Omega_{x}, \Omega_{y}, \omega\right)$ is some positive function that is smoothly changing for $q=\sqrt{\Omega_{x}^{2}+\Omega_{y}^{2}} \leq \frac{2 a}{F} \frac{\omega}{c_{0}}$ and sharply decreasing to zero for $q>\frac{2 a}{F} \frac{\omega}{c_{0}}$.

In order to obtain Fourier transform expressions of the transmit and receive radiation patterns, it is necessary to substitute the expression of $u_{0}^{(s)}$ into (15) and (21) for $u_{0}$, then calculate the Fourier transforms of receive-transmit radiation patterns $u^{(s)}$ according to (28).

It is difficult to obtain an analytical expression for $u^{(s)}$, thus it has been determined numerically. The function $u^{(s)}$ can be approximated in its common form as:

$$
\begin{aligned}
& u^{(s)}\left(\Omega_{x}, \Omega_{y}, \omega, z\right)=A^{(s)}\left(\sqrt{\Omega_{x}^{2}+\Omega_{y}^{2}}, \omega, z\right) \\
\times & \exp \left(i \psi_{1}(z-F) \psi_{2}\left(\frac{\Omega_{x}^{2}+\Omega_{y}^{2}}{2 \omega / c_{0}}\right)\right) \exp \left(i 2 \frac{\omega}{c_{0}} z\right),
\end{aligned}
$$

where the functions $\psi_{1}$ and $\psi_{2}$ are close to piecewise linear functions, and a positive function $A^{(s)}$ as a function of 


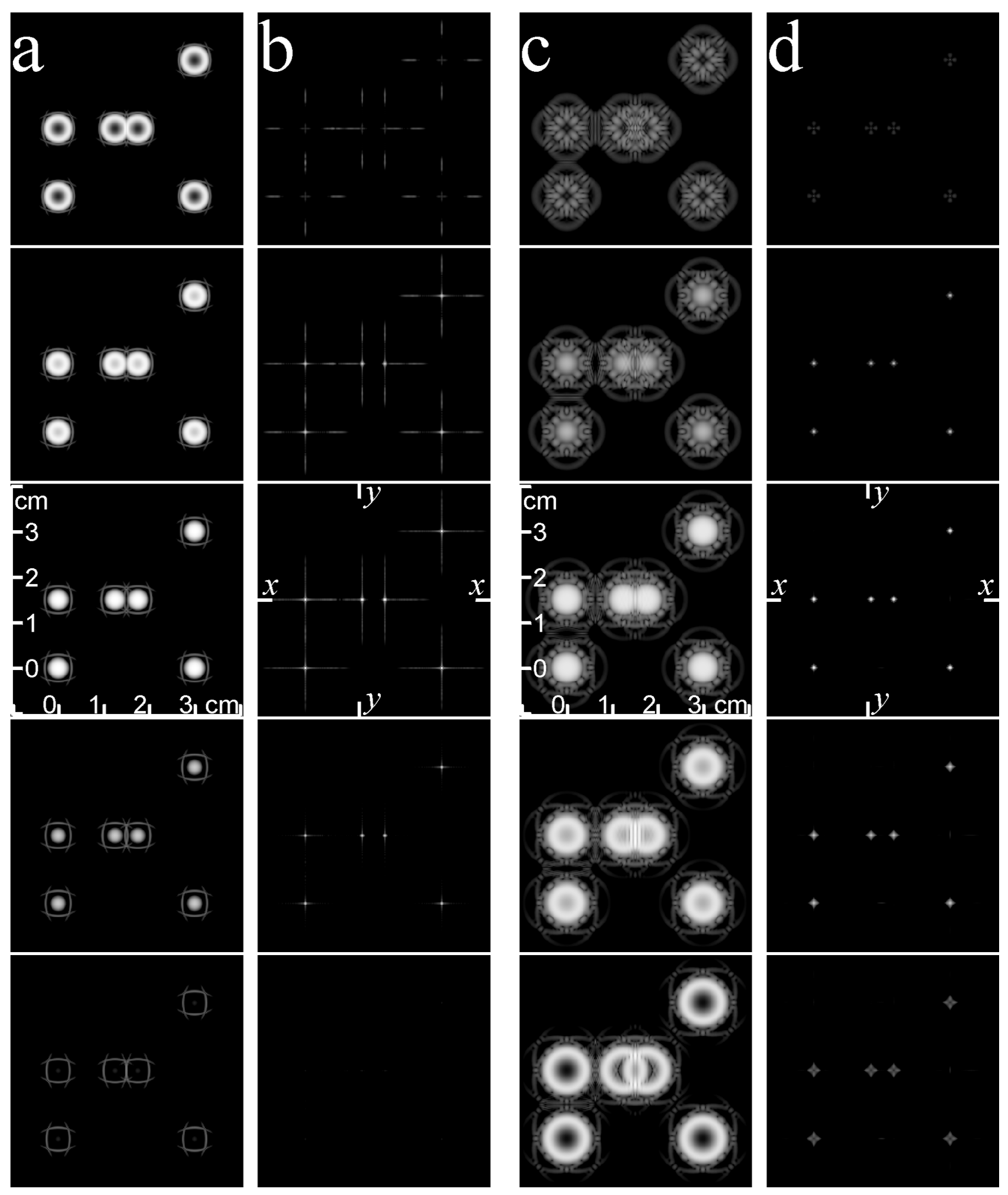

Fig. 3. The cross sections of 3-D image by the plane XY at various depths. (a)-(b) The signals are obtained from point reflectors located at the depth $0.3 \mathrm{~cm}$, (a) Before processing, (b) after processing, (c) and (d) the signals are obtained from point reflectors located at the depth $6.0 \mathrm{~cm}$, (c) before processing, (d) after processing. $F=1.8 \mathrm{~cm}, 2 a=1.2 \mathrm{~cm}, f=7.5 \mathrm{MHz}$; dynamic range 50 dB; cross sections step by depth $0.01 \mathrm{~cm}$. 


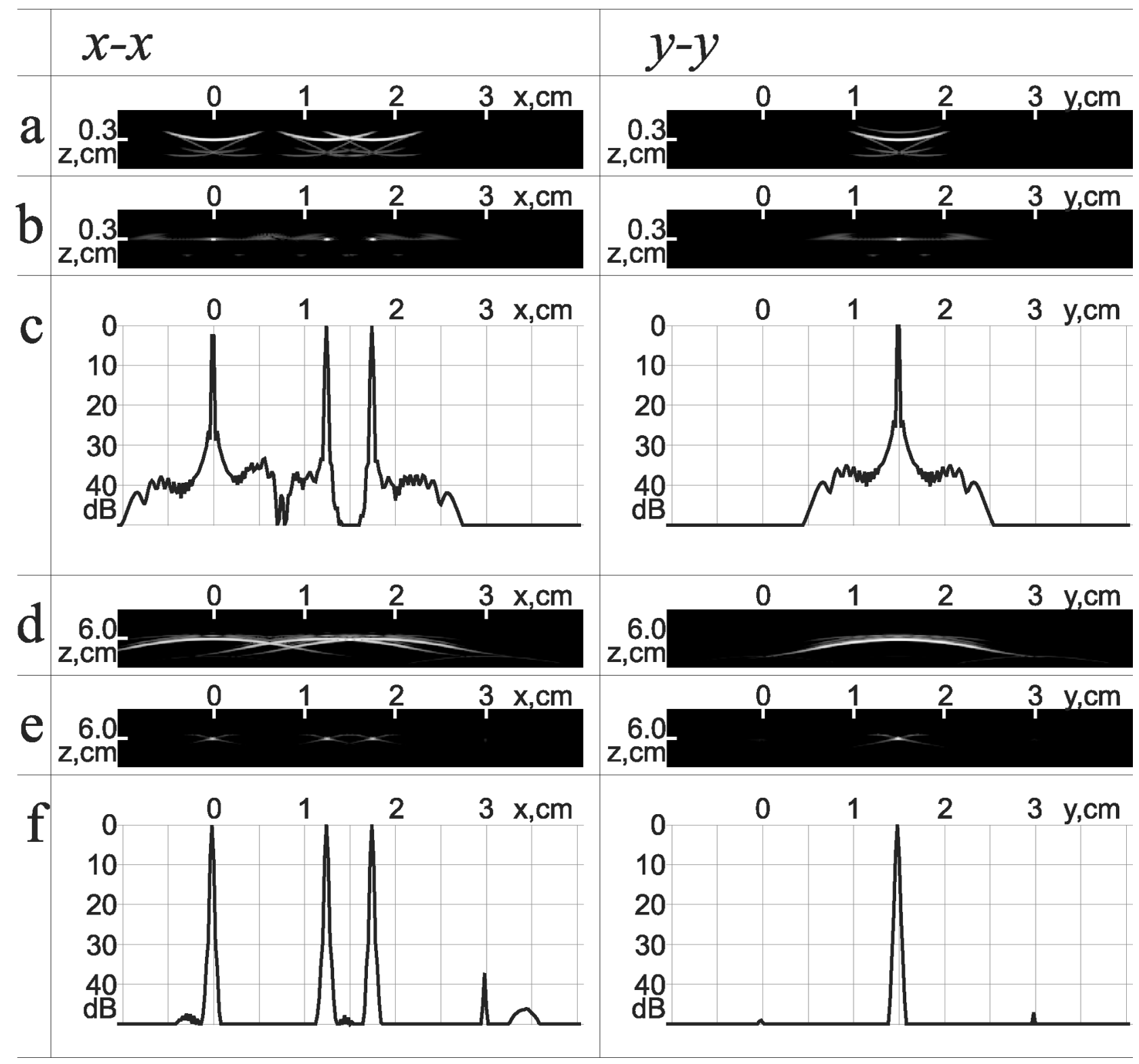

Fig. 4. The cross sections of 3-D image by the planes XZ and YZ and the signal magnitude. (a)-(c) The signals are obtained from point reflectors located at the depth $0.3 \mathrm{~cm}$. (a) Cross section of 3-D image before processing, (b) cross section of 3-D image after processing, (c) signal magnitude after processing. (d)-(f) The signals are obtained from point reflectors located at the depth $6.0 \mathrm{~cm}$. (d) Cross section of 3-D image before processing, (e) cross section of 3-D image after processing, (f) signal magnitude after processing. (x-x) XZ plane, ( $\mathrm{y}-\mathrm{y}$ ) YZ plane, $(\mathrm{x}-\mathrm{x})$ and $(\mathrm{y}-\mathrm{y})$ cross-sections positions are indicated by $\mathrm{x}-\mathrm{x}$ and $\mathrm{y}-\mathrm{y}$ lines on Fig. 3. The signals are the same as on Fig. 3. Dynamic range of the images is $50 \mathrm{~dB}$.

$\sqrt{\Omega_{x}^{2}+\Omega_{y}^{2}}$ changes from approximately rectangular at $z<$ $F$ to approximately Gaussian at $z>F$.

\section{B. Numerical Simulation}

The functions $A^{(s)}$ and $\varphi=\psi_{1} \cdot \psi_{2}$ are calculated for $F=8.0 \mathrm{~cm}, f=5 \mathrm{MHz}, 2 a=2.5 \mathrm{~cm}$, and for $F=2.0 \mathrm{~cm}$, $f=15 \mathrm{MHz}, 2 a=2.0 \mathrm{~cm}$. For $f=5 \mathrm{MHz}$ these functions are shown in Figs. 5 and 6 . In these figures the column "3D" corresponds to the transducer motion along both coordinates $x$ and $y$, and column " $2 \mathrm{D}$ " corresponds to the transducer motion along the coordinate $x$.
The width of the spatial spectrum is approximately unchanged in range from $z=0.1 \mathrm{~cm}$ to $z=12 \mathrm{~cm}$ for $f=5 \mathrm{MHz}$. This means that the bandwidth of the resulting radiation pattern and, consequently, the lateral resolution are practically the same at all the depths, including the region close to the aperture in front of the focal point. We apply the signal processing algorithm for the moving transducer, basically the same as in Section III-A. However, because the function $A^{(s)}$ is not smooth enough, and the functions $\psi_{1}$ and $\psi_{2}$ differ from linearity, we have to modify the algorithm as follows. The Fourier transform by time $t$ has to be performed for a few nearby intervals 


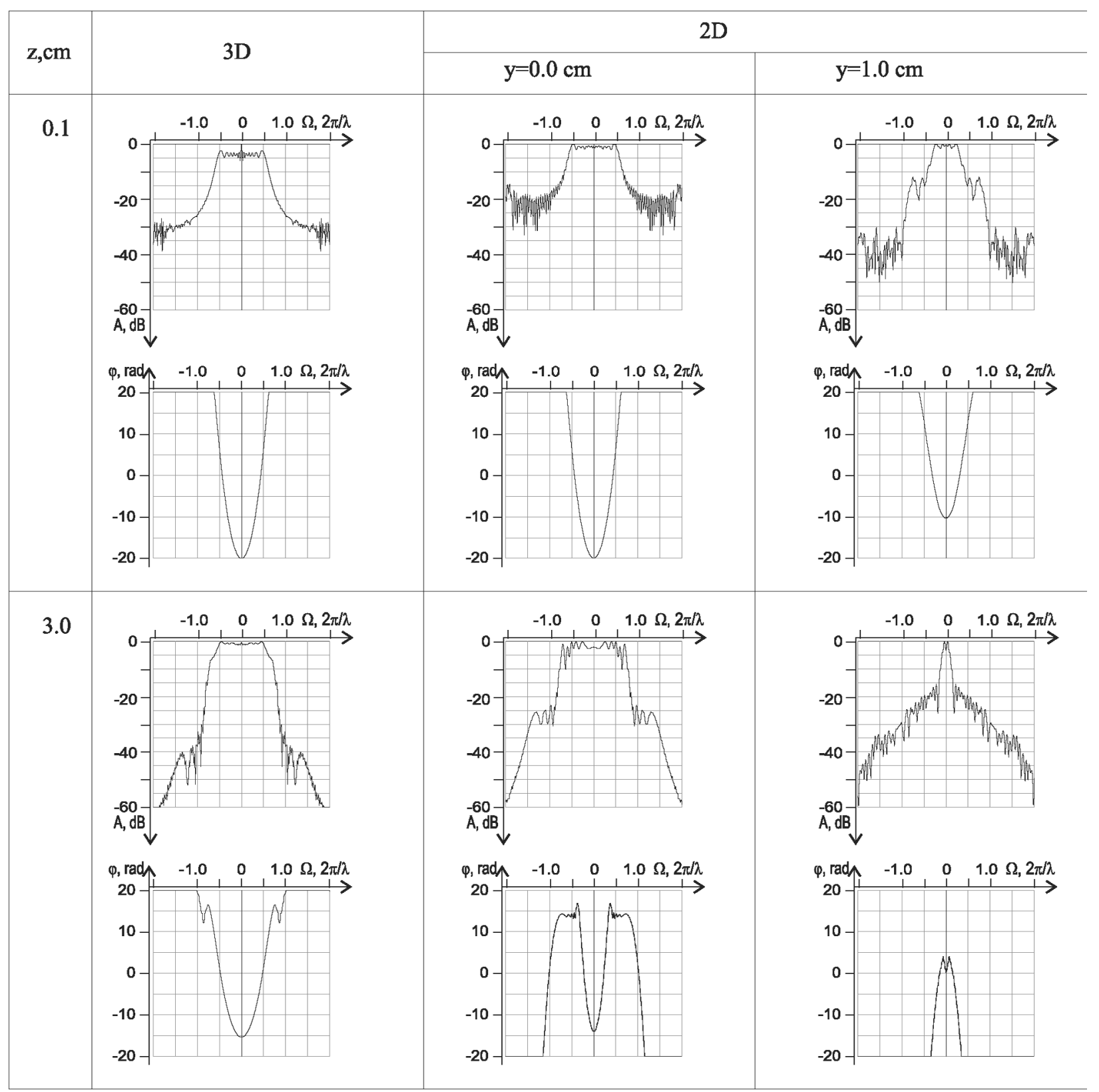

Fig. 5. The 2-D and 1-D signal spectra magnitude $A(\Omega)$ and phase $\varphi(\Omega)$ of spherical transducer radiation pattern in 3-D and 2-D cases. The 2-D-case spectra are given for different $y$-sections. $F=8.0 \mathrm{~cm}, 2 a=2.5 \mathrm{~cm}, f=5 \mathrm{MHz} . \Omega=\sqrt{\Omega_{x}^{2}+\Omega_{y}^{2}}$ for 3 -D column, $\Omega=\Omega_{x}$ for 2-D column. Depths $0.1 \mathrm{~cm}$ and $3.0 \mathrm{~cm}$.

of approximate size along the depth $z$ of $2 \mathrm{~cm}$ (see step two). For each of these intervals, a modified frequency $\tilde{\omega}$ [see (32)] is calculated by multiplying the second term in (32) by a factor that depends on the mean depth $z_{m}$ of the interval (thus taking into account the piecewise linearity of functions $\psi_{1}$ and $\psi_{2}$ ).

The inverse Fourier transform in step six is implemented for the data multiplied by an apodization function $W\left(\Omega_{x}, \Omega_{y}, z_{m}\right)$ that is approximately Gaussian. This function smoothes the function $A^{(s)}$ for the purpose of decreasing the side lobe levels in the resulting radiation pattern.

Tables I and II represent the width of radiation pattern and side lobe levels calculated for monochromatic signals and pulse signals with wide spectrum bands for carrier frequency $f$, focal distance $F$, aperture size $2 a$, and depth $z$. In Tables I and II, column 3-D corresponds to the transducer motion along both coordinates $x$ and $y$, and column 2-D corresponds to the transducer motion along coordinate $x$. The radiation pattern width is practically constant at all depths. The side lobe levels range from $-20 \mathrm{~dB}$ to $-60 \mathrm{~dB}$ for the monochromatic signal. For the pulse signals with wide spectrum brackets, the side lobe levels are lower and range from $-30 \mathrm{~dB}$ to $-60 \mathrm{~dB}$. All these data are obtained for 2-D scanning along coordinates $x$ and $y$. In [6]-[8] that deal with the investigation of different variants of the synthetic aperture technique, 1-D scanning of a spherical reflector along $x$-coordinate was considered. Be- 


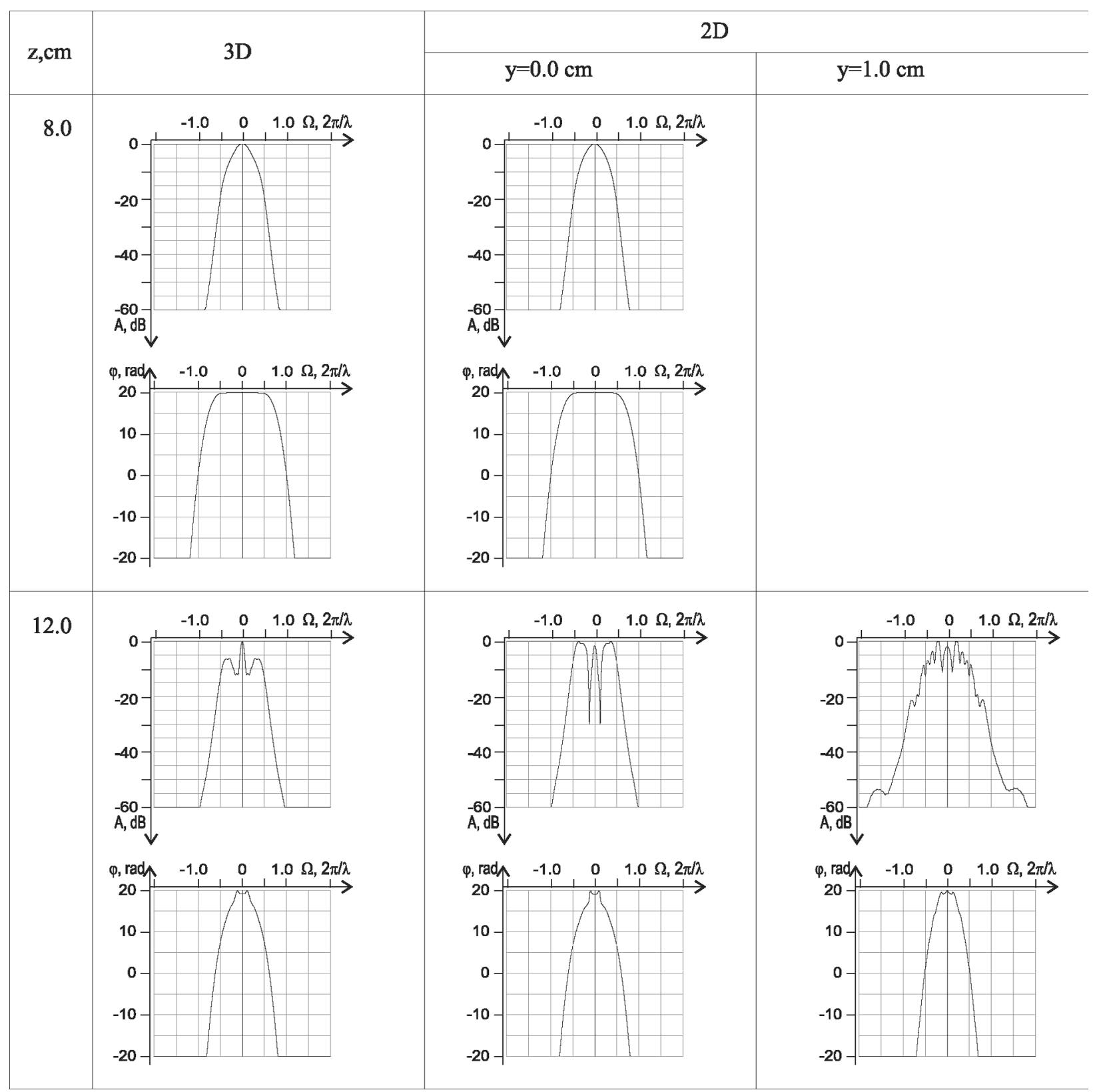

Fig. 6. The 2-D and 1-D signal spectra magnitude $A(\Omega)$ and phase $\varphi(\Omega)$ of spherical transducer radiation pattern in 3-D and 2-D cases. The 2-D-case spectra are given for different $y$-sections. $F=8.0 \mathrm{~cm}, 2 a=2.5 \mathrm{~cm}, f=5 \mathrm{MHz} . \Omega=\sqrt{\Omega_{x}^{2}+\Omega_{y}^{2}}$ for 3 -D column, $\Omega=\Omega_{x}$ for 2-D column. Depths $8.0 \mathrm{~cm}$ (at focus) and $12.0 \mathrm{~cm}$.

cause the function $u^{(s)}$ (37) depends upon the argument $\Omega_{x}^{2}+\Omega_{y}^{2}$ and in 1-D scanning it is possible to calculate only a function of $\Omega_{x}$, the diagram width increases as the transducer displaces along coordinate $y$, respectively, the point reflector. This reason also causes the increase in the side lobe levels. As a result of the mathematical simulation for input data given in Tables I and II, the radiation pattern width (or the beam width) has been increased two times (column 2-D). The side lobe levels varied in limits from $-5 \mathrm{~dB}$ to $-67 \mathrm{~dB}$ for the monochromatic signal, and from $-9 \mathrm{~dB}$ to $-69 \mathrm{~dB}$ for the wide-band signal. So, as one can see, in the near field the side lobes level is rather high due to the fact that the transducer moves only along one coordinate.

\section{Physical Experiment}

In order to verify these results, a physical experiment was conducted at the Bioacoustics Research Laboratory, University of Illinois, Urbana, IL. The positioning system has a positioning accuracy of $2 \mu \mathrm{m}$ (Daedal Inc., Harrison City, PA). The Parametric (Waltham, MA) spherical transducer had a center frequency of $5 \mathrm{MHz}$, bandwidth of $1.5 \mathrm{MHz}$, focal distance of $80 \mathrm{~mm}$, and aperture diameter of $25 \mathrm{~mm}$. A phantom (Model 539, ATS Laboratories, Inc., Bridgeport, CT) was placed in a degassed water tank $\left(22^{\circ} \mathrm{C}\right)$. The phantom is made of urethane rubber with a speed of sound of $1450 \mathrm{~m} / \mathrm{s}$ at room temperature and attenuation of $0.5 \mathrm{~dB} / \mathrm{cm} \cdot \mathrm{MHz}$. The embedded wire targets 
TABLE I

The Radiation Pattern Width (Diagram Width) and Side lobe Level (Side Lobes) After Signal Processing for Spherical Transducer.

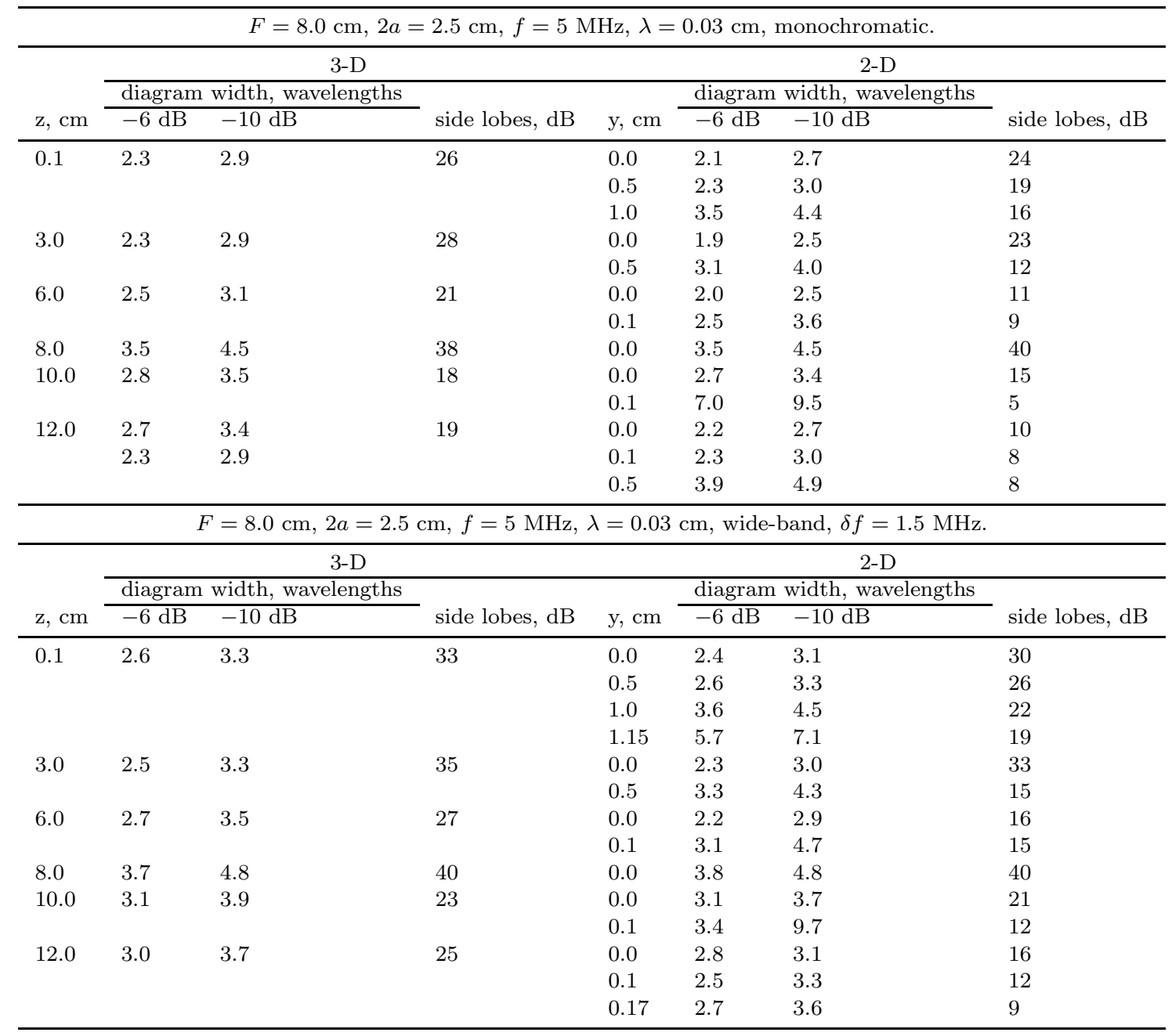

are made of monofilament nylon with $0.12 \mathrm{~mm}$ diameter. The cyst targets are anechoic regions with diameters of $1.5 \mathrm{~mm}$ to $6 \mathrm{~mm}$. The transducer was moved in only one direction along $x$-axis. A digitizing oscilloscope (LeCroy 9374L, Chestnut Ridge, NY) digitized the RF received signals with 8 bits. The sampling rate was $50 \mathrm{MHz}$. Data were collected on a SUN Sparc workstation, written on CD and processed on a personal computer with software that implemented the signal processing algorithm described in Section III-B.

Fig. 7 illustrates the phantom images before and after signal processing by the algorithm of Section III-B. A significant improvement of the lateral resolution and image contrast has been achieved. The lateral resolution is approximately the same in the zones in front and behind the focus, and corresponds to the theoretical estimation $\delta l=\frac{\lambda F}{2 a \cdot 1.5}$. The side lobe levels of order $-12 \mathrm{~dB}$ to $-18 \mathrm{~dB}$ also match the estimates that are based on the results of the mathematical simulation for 1-D scanning.

In order to study the influence of the $y$ coordinate, the Fourier transforms of the RF signals from the wire reflec- tors in the near field were corrected by the mathematical model that takes into account the scattering along the $y$-coordinate. The images of the wire reflectors near the phantom surface are shown on Fig. 8. Taking into account the preliminary knowledge of the wire reflector geometry at the signal processing allows to decrease the side lobes level, namely, the side lobe level has been decreased to $-18 \mathrm{~dB}$.

\section{InVESTigation of Rectangular TRAnsducer}

\section{A. Theory}

Transducers with a rectangular aperture are widely used. Therefore, consider a rectangular aperture transducer moving along Cartesian coordinates $x$ and $y$ in the $z=0$ plane, as was done in Section IV-A for spherical transducer.

It is assumed that a thin rectangular lens with a fixed focal distance $F$ is installed on the active transducer surface, and the transducer is excited by a spatially homogeneous 
TABLE II

The Radiation Pattern Width (Diagram Width) and Side Lobe Level (Side Lobes) After Signal Processing for Spherical TransDucer.

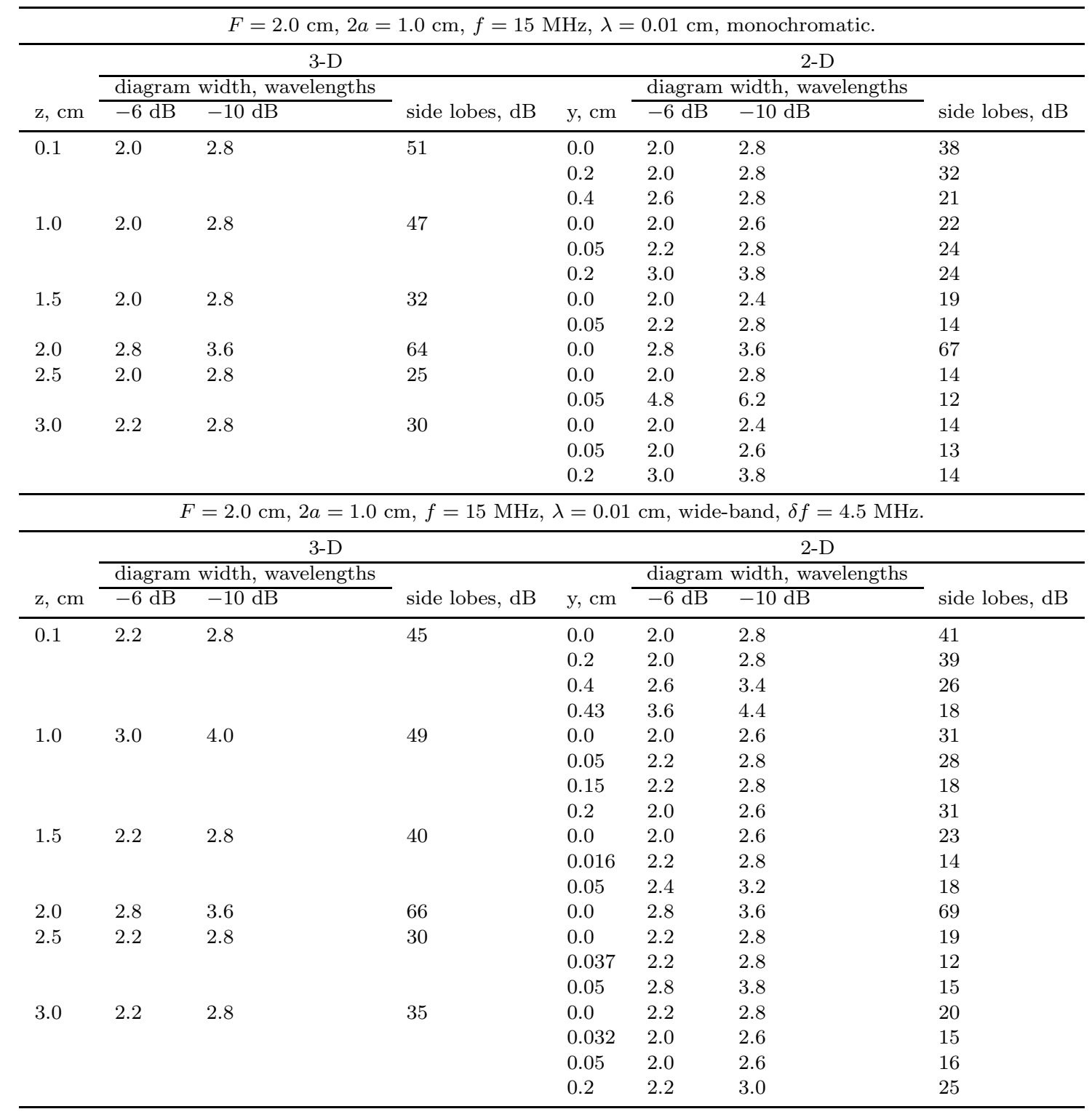

displacement along the $z$-axis. The vibrating velocity $V_{1}$ at frequency $\omega$ is determined by (34). For the rectangular aperture, the Fourier transform of exponential term in (34) is expressed by:

$$
\begin{array}{r}
u_{0}^{(r)}\left(\Omega_{x}, \Omega_{y}, \omega\right)=\int_{-a_{x}}^{a_{x}} \int_{-a_{y}}^{a_{y}} \exp \left(-i\left(\Omega_{x} \xi+\Omega_{y} \eta\right)\right) \\
\exp \left(-i \frac{\omega}{c_{0}} \frac{\xi^{2}+\eta^{2}}{2 F}\right) d \xi d \eta \\
\quad=u_{0}^{(r, x)}\left(\Omega_{x}, \omega\right) u_{0}^{(r, y)}\left(\Omega_{y}, \omega\right),
\end{array}
$$

where $2 a_{x}$ and $2 a_{y}$ are the sizes of the aperture along the $x$ and $y$ axes, and where

$$
\begin{aligned}
u_{0}^{(r, x)}\left(\Omega_{x}, \omega\right) & =\int_{-a_{x}}^{a_{x}} \exp \left(-i \Omega_{x} \xi\right) \exp \left(-i \frac{\omega}{c_{0}} \frac{\xi^{2}}{2 F}\right) d \xi, \\
u_{0}^{(r, y)}\left(\Omega_{y}, \omega\right) & =\int_{-a_{y}}^{a_{y}} \exp \left(-i \Omega_{y} \eta\right) \exp \left(-i \frac{\omega}{c_{0}} \frac{\eta^{2}}{2 F}\right) d \eta,
\end{aligned}
$$

Thus, the 2-D Fourier transform of the rectangular aperture function is a product of two 1-D Fourier transforms. The formula for any of these functions can be presented as:

$$
u_{0}^{(r, g)}=A_{0}^{(g)}\left(\Omega_{g}, \omega\right) \exp \left(-\frac{i \Omega_{g}^{2} F}{2 \omega / c_{0}}\right),
$$



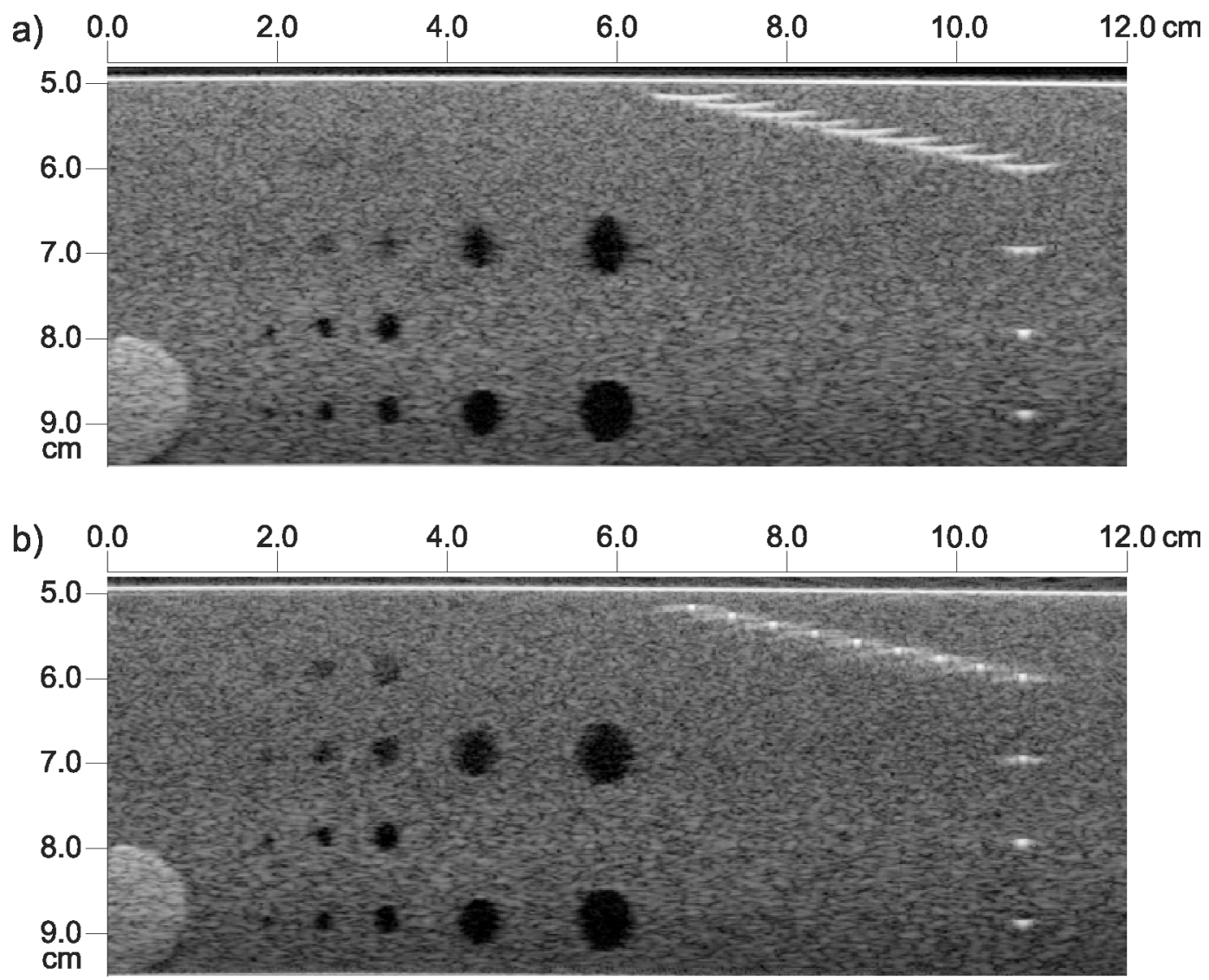

Fig. 7. The image of phantom before and after signal processing for moving spherical transducer. $F=8.0 \mathrm{~cm}, 2 a=2.5 \mathrm{~cm}, f=5 \mathrm{MHz}$. Dynamic range $70 \mathrm{~dB}$. (a) Before signal processing, (b) after signal processing.

where index $g$ is $x$ or $y$ and a function $A_{0}^{(g)}$ is some positive function that smoothly varies for $\left|\Omega_{g}\right| \leq \frac{2 a_{g} \omega}{F c_{0}}$ and sharply decreases at larger values of $\left|\Omega_{g}\right|$.

Similarly (see Section IV-A), to obtain the transmit and receive radiation patterns' Fourier transforms, it is necessary to multiply (38) by a corresponding Fourier transforms with the free-space spatial impulse response. It is assumed that $\left|\Omega_{g}\right|_{m}<0.6 \frac{\omega}{c_{0}}$. In this case the Fourier transform can be approximately presented as:

$$
\begin{aligned}
& H_{f r}\left(\Omega_{x}, \Omega_{y}\right)=\exp \left(i z \sqrt{\left(\frac{\omega}{c_{0}}\right)^{2}-\Omega_{x}^{2}-\Omega_{y}^{2}}\right) \\
& \approx \exp \left(i z \frac{\omega}{c_{0}}\right) \exp \left(-i z \frac{\Omega_{x}^{2}}{2 \omega / c_{0}}\right) \exp \left(-i z \frac{\Omega_{y}^{2}}{2 \omega / c_{0}}\right)
\end{aligned}
$$

where $H_{f r}$ is a product of two similar functions of $\Omega_{x}$ and $\Omega_{y}$. That is why, taking into account (41), we get that the Fourier transform of the resulting receive-transmit radiation pattern, namely:

$$
u^{(r)}\left(\Omega_{x}, \Omega_{y}, \omega, z\right)=u^{(r, x)}\left(\Omega_{x}, \omega, z\right) u^{(r, y)}\left(\Omega_{y}, \omega, z\right),
$$

where

$$
\begin{aligned}
& u^{(r, g)}\left(\Omega_{g}, \omega, z\right)=A^{(r, g)}\left(\Omega_{g}, \omega, z\right) \\
& \quad \exp \left(i \psi_{1}^{(g)}(z-F) \psi_{2}\left(\frac{\Omega_{g}^{2}}{2 \omega / c_{0}}\right)\right) \exp \left(i \frac{\omega}{c_{0}} z\right),
\end{aligned}
$$

( $g$ is $x$ or $y$ ).

Formula (44) is an approximate expression, having been obtained by means of an analytical estimation and proved by numerical simulation; $\psi_{1}^{(g)}$ and $\psi_{2}^{(g)}$ are close to piecewise linear functions.

\section{B. Computer Simulation}

For comparison with the spherical transducer case, a computer simulation for the square aperture was conducted with the same initial data. The same signal processing algorithm (see Section IV-B) was applied. The results of the simulation showed that the Fourier transform of the radiation pattern $u^{(r)}$ is presented with sufficient accuracy by (43), and correspondingly different parameters of transducer can be chosen along axes $x$ and $y$. Fig. 9 shows the graphs of functions $A^{(r, x)}$ and $\psi_{1}^{(x)}(z-F) \psi_{2}^{(x)}\left(\frac{\Omega_{x}^{2}}{2 \omega / c_{0}}\right)$ calculated for $F=8.0 \mathrm{~cm}, f=5 \mathrm{MHz}, 2 a_{x}=2.5 \mathrm{~cm}$. The width of the spatial spectrum $A^{(r, x)}$ does not substantially change in the interval of depths from $z=0.1 \mathrm{~cm}$ 


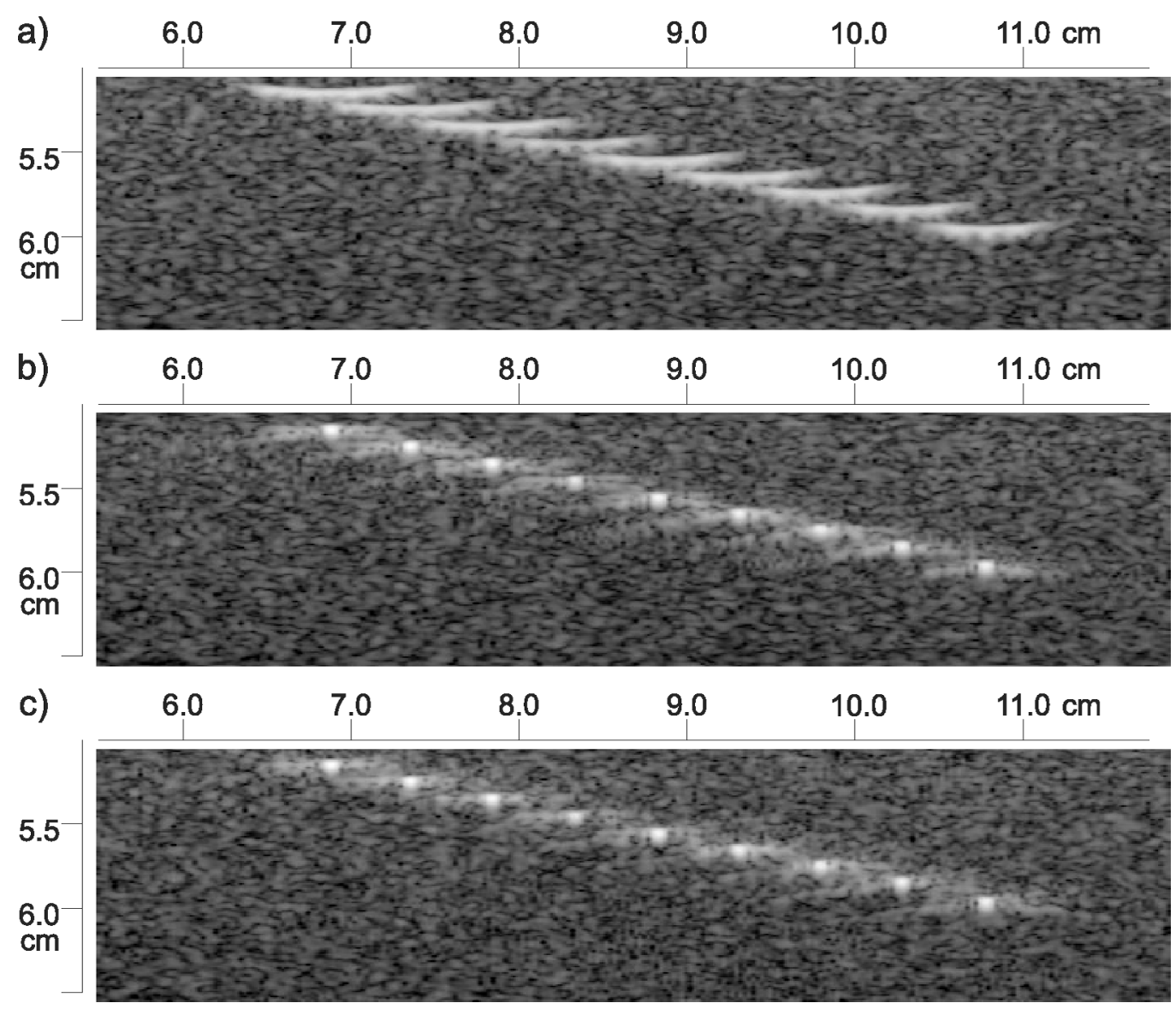

Fig. 8. The image of phantom in front of the focus before and after signal processing, and after signal processing with y-coordinate taken into account. $F=8.0 \mathrm{~cm}, 2 a=2.5 \mathrm{~cm}, f=5 \mathrm{MHz}$. Dynamic range $60 \mathrm{~dB}$. (a) Before processing, (b) after processing, (c) after processing with y-coordinate taken into account.

to $z=12 \mathrm{~cm}$. The lateral resolution does not change over the entire range of depths $z$. Table III presents the values of the radiation pattern width and the side lobe levels calculated for monochromatic signals and pulse signals with large bandwidth for the center frequency $f=5 \mathrm{MHz}$, focal distance $F=8.0 \mathrm{~cm}$, aperture size $2 a=2.5 \mathrm{~cm}$, and for various values of the depth $z$. The column " 3 D" corresponds to the transducer motion along both coordinates $x$ and $y$, and column "2-D" corresponds to the transducer motion along the coordinate $x$. The radiation pattern width is practically constant at all depths. The side lobe levels change from $-15 \mathrm{~dB}$ to $-30 \mathrm{~dB}$ for the monochromatic signal and do not depend upon displacement of the point reflector along $y$-axis. For the pulse signal with large bandwidth, the side lobe levels range from $-17 \mathrm{~dB}$ to $-38 \mathrm{~dB}$.

In addition, a numerical simulation of the signal processing algorithm was carried out for a phased array with a rectangular aperture moved along the $y$-coordinate. Along the longitudinal coordinate of the array, traditional dynamic focusing was implemented with a cut function of Gaussian apodization.

A thin cylindrical convex lens with a focal distance $F=3.0 \mathrm{~cm}$ and aperture size $2 a_{y}=0.6 \mathrm{~cm}$ was installed at the array surface. For the $y$-coordinate, the algorithm's radiation pattern synthesis was applied for the case of a transducer moving along the $y$-coordinate. In Table IV the values of the radiation patterns' widths and the side lobe levels along the $x$ and $y$ coordinates are given. The radiation pattern width along the $x$-coordinate changes from $0.036 \mathrm{~cm}$ to $0.244 \mathrm{~cm}$, depending on the depth $z$; and the width along $y$-coordinate varies within a small interval from $0.176 \mathrm{~cm}$ to $0.290 \mathrm{~cm}$. The side lobe levels are practically constant and equal to $-40 \mathrm{~dB}$.

\section{Physical Experiment}

For testing these results, we used digitized data from a phased array obtained at Biomedical Ultrasonics Laboratory, University of Michigan (http://bul.eecs.umich.edu). These data were processed by means of the algorithm from Section IV-B for a rectangular transducer moving only along the $x$-axis. The primary data were transformed in a way that allowed modeling the rectangular transducer with aperture of size $2 a_{x}=0.6 \mathrm{~cm}$ and a fixed focal distance $F=3.0 \mathrm{~cm}$. The total length of the scanning trajectory was equal to the phased array length of $2.8 \mathrm{~cm}$. Fig. 10 illustrates the simulation results from the phantom with point reflectors placed at various depths $z$ and for the phantom with cysts. As one can see from Fig. 10, 
TABLE III

The Radiation Pattern Width (Diagram Width) and Side Lobe Level (Side Lobes) After Signal Processing for Square TRANSDUCER.

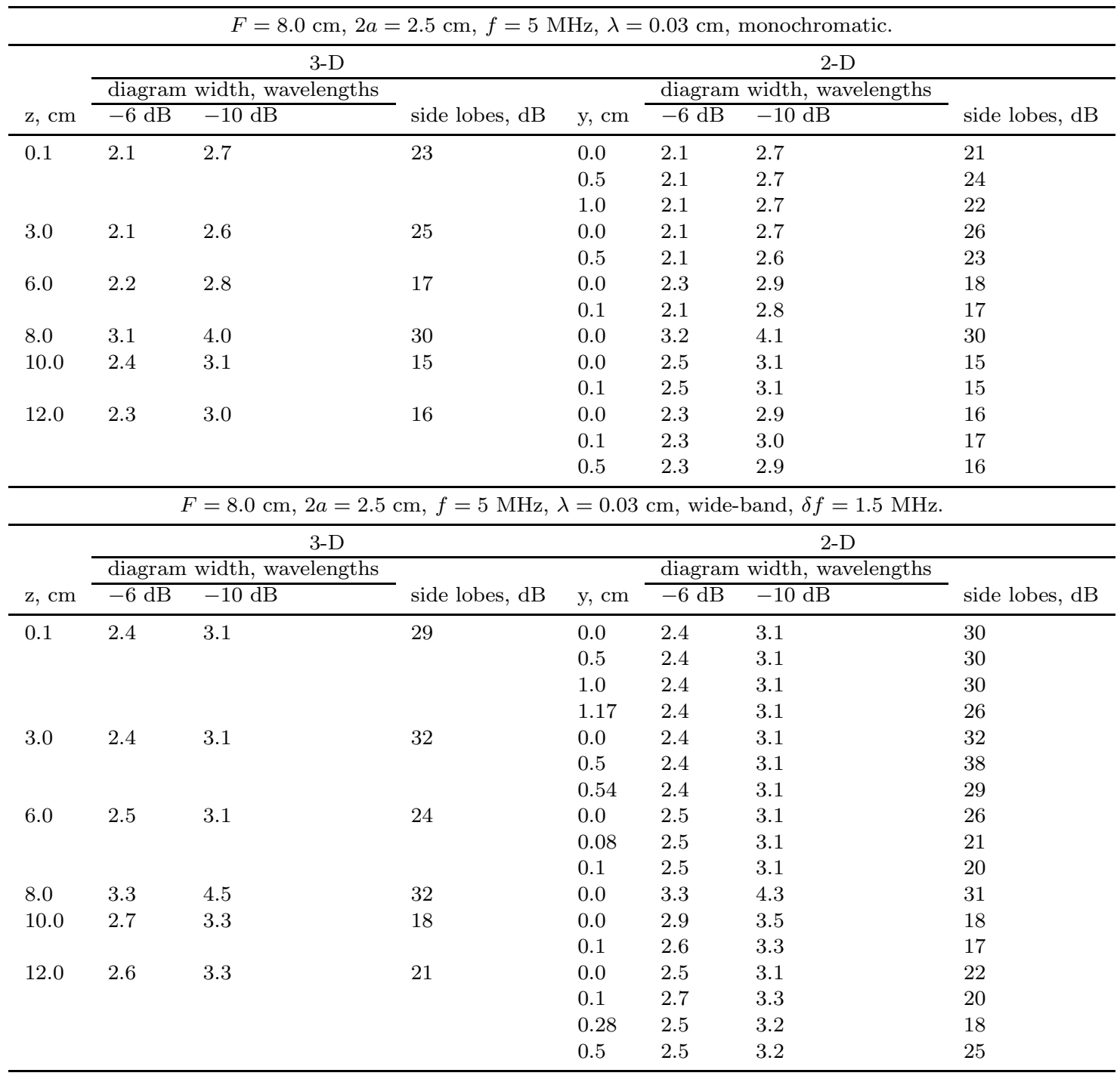

TABLE IV

The Radiation Pattern Width (Diagram Width) and Side Lobe Level (Side Lobes) After Signal Processing for Phased Array with Rectangular Surface Moving Along $y$-Axis.

\begin{tabular}{|c|c|c|c|c|c|c|c|}
\hline \multirow[b]{3}{*}{$\mathrm{z}, \mathrm{cm}$} & \multicolumn{4}{|c|}{$\mathrm{X}$} & \multicolumn{3}{|c|}{$\mathrm{Y}$} \\
\hline & \multicolumn{2}{|c|}{ before processing } & \multicolumn{2}{|c|}{ after processing } & \multirow{2}{*}{$\begin{array}{l}\text { before processing } \\
\text { diagram width*, } \\
\text { wavelengths }\end{array}$} & \multicolumn{2}{|c|}{ after processing } \\
\hline & $\begin{array}{l}\text { diagram width*, } \\
\text { wavelengths }\end{array}$ & $\begin{array}{l}\text { side lobes, } \\
\mathrm{dB}\end{array}$ & $\begin{array}{l}\text { diagram width*, } \\
\text { wavelengths }\end{array}$ & $\begin{array}{l}\text { sidelobes, } \\
\mathrm{dB}\end{array}$ & & $\begin{array}{l}\text { diagram width*, } \\
\text { wavelengths }\end{array}$ & $\begin{array}{l}\text { side lobes, } \\
\text { dB }\end{array}$ \\
\hline 0.1 & 0.7 & 35 & 0.6 & 41 & 11.2 & 3.5 & 31 \\
\hline 3.0 & 1.2 & 39 & 1.2 & 39 & 4.8 & 4.8 & 43 \\
\hline 6.0 & 2.4 & 41 & 2.5 & 41 & 10.2 & 5.6 & 41 \\
\hline 8.0 & 3.2 & 38 & 3.3 & 41 & 14.6 & 5.8 & 42 \\
\hline 10.0 & 4.0 & 37 & 4.1 & 42 & 18.0 & 5.8 & 43 \\
\hline 12.0 & 4.9 & 33 & 4.9 & 42 & 24.2 & 5.8 & 44 \\
\hline
\end{tabular}

* Diagram width at $-10 \mathrm{~dB}$ level 


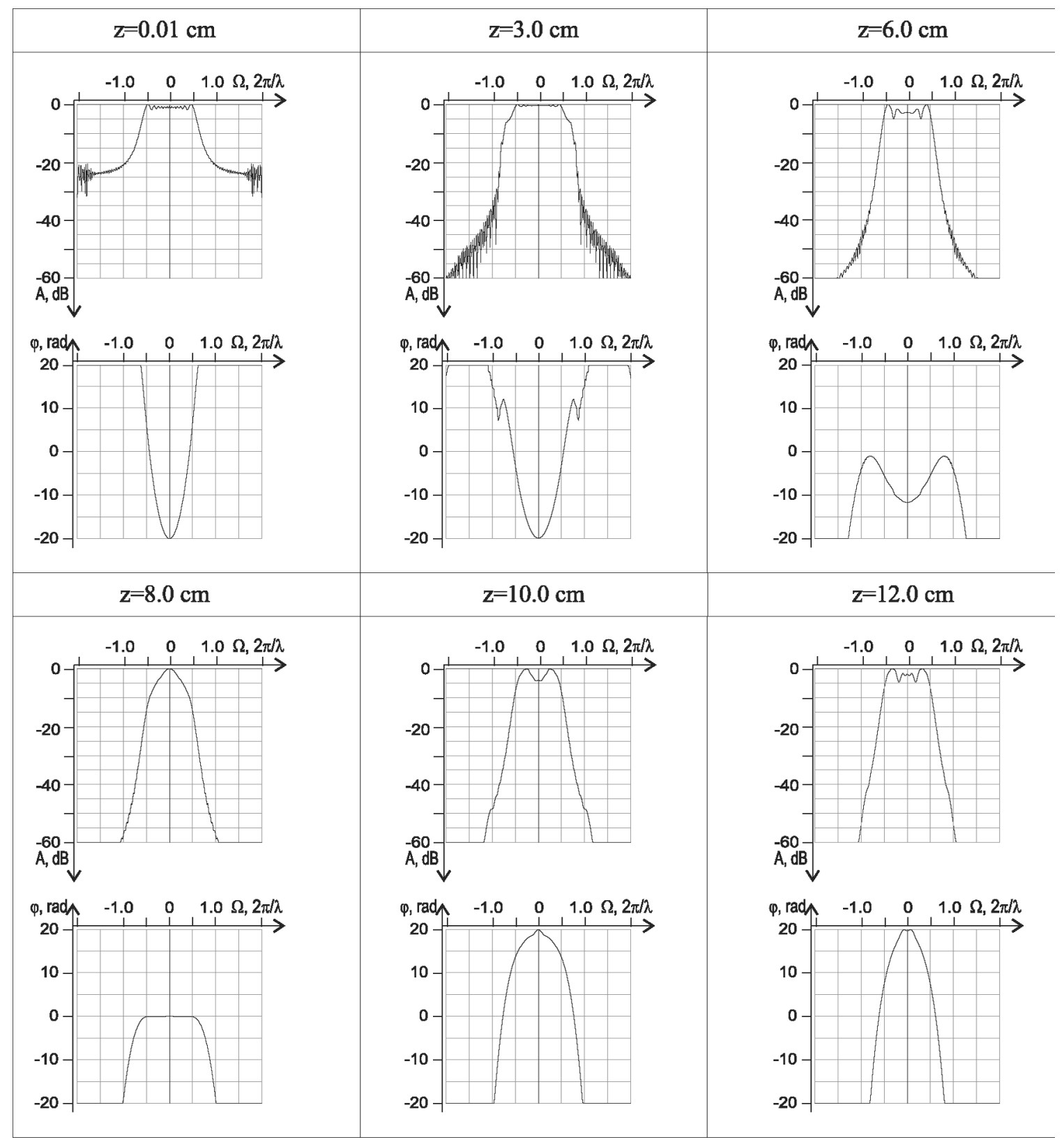

Fig. 9. The spectrum magnitude $A\left(\Omega_{x}\right)$ and phase $\varphi\left(\Omega_{x}\right)$ of square transducer radiation pattern. $F=8.0 \mathrm{~cm}, 2 a=2.5 \mathrm{~cm}, f=5 \mathrm{MHz}$.

after signal processing, the point reflectors' widths do not substantially change with the depth while the contrast of cysts increases. The pattern diagram width is about $0.24 \mathrm{~cm}$, which is approximately equal to the theoretical estimations value.

\section{Estimation of the Effect of Scattering OBJECTS Motion}

The results presented herein were obtained under the assumption that the objects that scatter sound do not move. If these objects move, the synthesized radiation pattern will change. These changes are affected by the axial component $v_{z}$ of the scatterer velocity vector and its lateral components $\left(v_{x}, v_{y}\right)$.
In order to estimate the effect of moving scatterers, we neglect the receive signal component of the dipole scattering in (19) and represent it as a sum of an array of point reflectors with the coordinates $\left(x_{n}, y_{n}, z_{n}\right)$ with reflection coefficient $\gamma_{n}$ :

$$
V_{r e}(x, y, z)=K_{6}(\omega) \sum_{n} L\left(x-x_{n}, y-y_{n}, z_{n}, \omega\right) \gamma_{n},
$$

where $L\left(x-x_{n}, y-y_{n}, z_{n}, \omega\right)=L_{t r}\left(x-x_{n}, y-\right.$ $\left.y_{n}, z_{n}, \omega\right) \cdot L_{r e}\left(x-x_{n}, y-y_{n}, z_{n}, \omega\right)$ is a product of the transmit and receive radiation patterns; $x, y$ are the coordinates of moving transducer; and $K_{6}$ is a scale function. Taking into account the character of (31), the function $L$ can be presented as: 


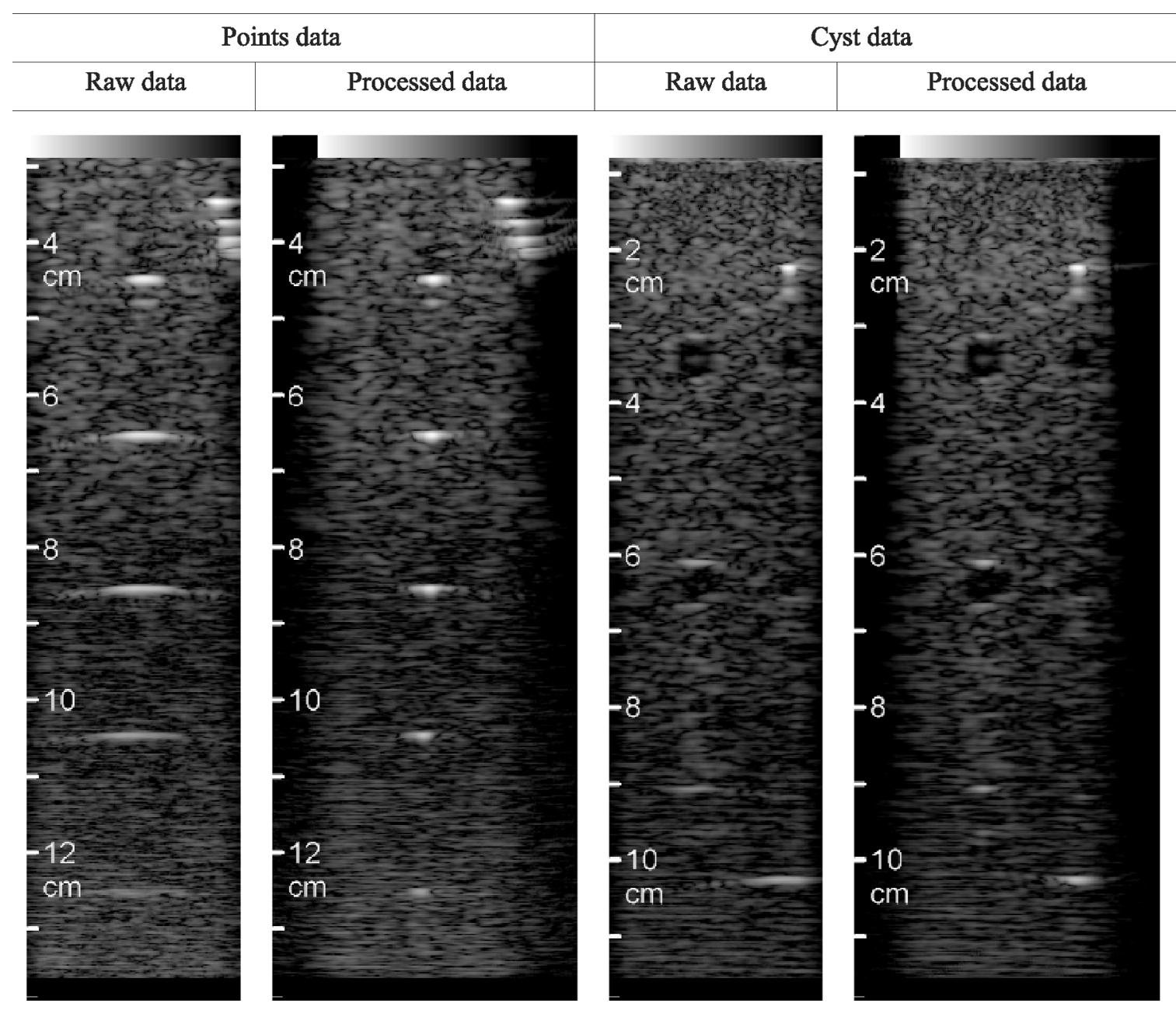

Fig. 10. The image of phantom before and after signal processing for moving rectangular transducer. $F=3.0 \mathrm{~cm}, 2 a=0.6 \mathrm{~cm}, f=3.3 \mathrm{MHz}$. Dynamic range $50 \mathrm{~dB}$.

$$
L(x, y, z, \omega)=\hat{L}(x, y, z, \omega) \exp \left(i \frac{2 \omega}{c_{0}} z\right),
$$

where $\hat{L}$ characterizes a complex function determining width of the radiation pattern at distance $z$ (before processing). Let us represent the Fourier transform of the function $\hat{L}$ by coordinates $x, y$ in the form:

$$
\begin{aligned}
& u_{\hat{L}}\left(\Omega_{x}, \Omega_{y}, \omega, z\right)= \\
& \int \hat{L}(x, y, z, \omega) \exp \left(-i\left(\Omega_{x} x+\Omega_{y} y\right)\right) d x d y \\
& \quad=\hat{A}\left(\Omega_{x}, \Omega_{y}, z, \omega\right) \exp \left(-i \frac{\zeta}{2}\left(\Omega_{x}^{2}+\Omega_{y}^{2}\right)\right),
\end{aligned}
$$

where, $\zeta$ is calculated in correspondence with (26). The inverse Fourier transform of the function $\hat{A}$ by $\Omega_{x}$ and $\Omega_{y}$ with the same apodization function $W$ (Section IV-B) characterizes the synthesized radiation pattern.

Let us denote the components of the scanning velocity along the axes $x$ and $y$ by $v_{s, x}$ and $v_{s, y}$, respectively. We suppose that $\left|v_{x}\right|,\left|v_{y}\right|,\left|v_{s}\right| \ll \min \left\{\left|v_{s, x}\right|, \mid v_{s, x}\right\}$. We denote as $t$ the time of the scanning process, $-\frac{T}{2}<t \leq \frac{T}{2}$, where $T$ is the scanning time interval. Consider that the transducer moves along the $y$-axis discretely, with a step $\delta y$ with time intervals $T_{0}$, and for the time $T_{0}$ the transducer moves along the $x$-axis a distance $X_{0}$. In order to achieve a constant lateral resolution at all the depths $z$, the value of $X_{0}$ must be approximately equal to the maximum width of the radiation pattern $L$ along depth $z$. Taking into account these assumptions, we can put down the coordinates of the transducer's center at the moment $t$ at $m T_{0}<t \leq(m+1) T_{0}\left(m=\frac{T}{2 T_{0}}, \ldots, 0, \ldots, \frac{T}{2 T_{0}}\right)$ as:

$$
x=v_{s, x}\left(t-m T_{0}\right), y=v_{s, y} t .
$$

At time $t$ for the $n$-th reflector moving with the velocity vector $\left(v_{x}, v_{y}, v_{z}\right)$, we present the received signal by:

$$
\begin{array}{r}
\tilde{V}_{r e}^{(n)}(x, y, \omega)=K_{6} \hat{L}\left(v_{s, x}\left(t-m T_{0}\right)-\left(x_{n}+v_{x} t\right),\right. \\
\left.v_{s, y} t-\left(y_{n}-v_{y} t\right), z_{n}+v_{z} t, \omega\right) \\
\times \exp \left(\frac{2 i \omega}{c_{0}}\left(z_{n}+v_{z} t\right)\right) .
\end{array}
$$

Performing a Fourier transform of (49) by $x=v_{s, x}(t-$ $\left.m T_{0}\right)$ and $y=v_{s, y} t$ with step $\delta \Omega_{x}=\frac{2 \pi}{v_{s, x} T_{0}}$, we obtain: 


$$
\begin{aligned}
\hat{V}_{r e}^{(n)}\left(\Omega_{x}, \Omega_{y}, \omega\right)=u_{\hat{L}}\left(\Omega_{x}\left(1-\frac{v_{x}}{v_{s, x}}\right)\right. & \\
- & \left.\frac{2 v_{z}}{v_{s, x}} \frac{\omega}{c_{0}}, \Omega_{y}\left(1-\frac{v_{y}}{v_{s, y}}\right)-\frac{2 v_{z}}{v_{s, y}} \frac{\omega}{c_{0}}, z_{n}\right) \\
& \times \exp \left(i\left(\Omega_{x} x_{n}+\Omega_{y} y_{n}\right)\right) \exp \left(i \frac{2 \omega}{c_{0}} z_{n}\right),
\end{aligned}
$$

where $u_{\hat{L}}$ is a Fourier transform of the radiation pattern $\hat{L}$.

From (50) it follows that the lateral components of the reflector's velocity $v_{x}, v_{y}$ lead to the change of a scale of the function $u_{\hat{L}}$ by $\Omega_{x}, \Omega_{y}$, and, consequently, mainly cause broadening or narrowing of radiation pattern that is proportional to $\left(1-\frac{v_{x}}{v_{s, x}}\right)^{-1}$ along the $x$-axis and to $\left(1-\frac{v_{y}}{v_{s, y}}\right)^{-1}$ along the $y$-axis. The component of the velocity $v_{z}$ leads to the frequency shifts of $\Omega_{x}, \Omega_{y}$ by $\frac{2 v_{z}}{v_{s, x}} \frac{\omega}{c_{0}}$, $\frac{2 v_{z}}{v_{s, y}} \frac{\omega}{c_{0}}$. Consequently, when taking into account the focusing term $\exp \left(i \frac{\zeta\left(\Omega_{x}^{2}+\Omega_{y}^{2}\right)}{2}\right)$ and (26) in the signal processing algorithm, it leads to the shift of the radiation patterns along $x$ and $y$ axes by:

$$
\begin{aligned}
& \Delta x=\frac{\zeta}{2} \frac{4 \omega}{c_{0}} \frac{v_{z}}{v_{s, x}} \approx \frac{\left(z_{n}-F\right) v_{z}}{v_{s, x}} \\
& \Delta y=\frac{\zeta}{2} \frac{4 \omega}{c_{0}} \frac{v_{z}}{v_{x, y}} \approx \frac{\left(z_{n}-F\right) v_{z}}{v_{s, y}} .
\end{aligned}
$$

The greater the depth of scatter is, more significant this shift is.

It should be noted that, as the maximum depth increases, the ultrasound propagation path increases and the maximum scanning velocities decrease.

For the purpose of an abdominal examination, it is reasonable to use a linear array with a characteristic maximum depth of about $15 \mathrm{~cm}$. The size of this array is of the order of $6 \mathrm{~cm}$ along the axis of electronic scanning $(x$-axis) and is about $0.6 \mathrm{~cm}$ along the perpendicular size (y-axis). The array has a fixed focal distance $F=3 \mathrm{~cm}$ along the $y$-axis. In the $x$-axis plane, the signals should be transmitted and received simultaneously for all elements of the linear array. For the signal processing in the $x$-axis plane, we choose the size of receiving active aperture of $2 a=2 \mathrm{~cm}$ and focal distance $F=3 \mathrm{~cm}$. For each value of $x$-coordinate of the linear array, the signals of the active subaperture are calculated with the same coordinate of its center. These signals are calculated using the signals received by the array elements contained in this subaperture.

In order to obtain a 3-D image, an array should be moved along the $y$-axis a distance of $5 \mathrm{~cm}$. All the received signals of subapertures then are processed using the algorithms of Section III-B. The total scanning time will be of order 0.02 second and the corresponding velocity of movement $v_{s, y}=250 \mathrm{~cm} /$ second. In this case (50) should be used only for one lateral frequency $\Omega_{y}$. If the maximum velocity of a scatterer is estimated to be $v=5 \mathrm{~cm} / \mathrm{second}$, the radiation pattern broadening will be characterized by factor 1.02 and the maximum shift $\Delta y=0.28 \mathrm{~cm}$, that is, approximately equal to the lateral resolution along $y$-axis for $f=3 \mathrm{MHz}$ (see Section $\mathrm{V}$ ).

In the case of biomicroscopy, the maximum value of depth is about $4 \mathrm{~mm}$ [19]. If one applies a spherical transducer with diameter $D=1.5 \mathrm{~mm}$, carrier frequency $f=50 \mathrm{MHz}$ and fixed focal distance $F=2 \mathrm{~mm}$, then to obtain a 3 -D image in the volume $1.5 \mathrm{~mm} \times 6 \mathrm{~mm} \times 5 \mathrm{~mm}$ the total time of moving along $x$ - and $y$-coordinates will be of the order of 0.05 second. The scanning velocity along the $y$-axis will be about $100 \mathrm{~mm} /$ second. It follows from (51) that the maximum shift $\Delta y=0.02 \mathrm{~mm}$ at the scatterer velocity $v_{z}=1 \mathrm{~mm} / \mathrm{second}$. The lateral resolution along $x$ - and $y$-axes will be of order $0.06 \mathrm{~mm}$, and the axial resolution along $z$-coordinate will be $0.01 \mathrm{~mm}$ at the frequency bandwidth $\Delta f=40 \mathrm{MHz}$. These estimations show that the mechanical moving of the transducer along both coordinates is acceptable for low scatterer velocity.

For nondestructive evaluation applications, the velocities of the scatterers are very small. Thus, it is possible to apply the mechanical moving of transducer along both coordinates.

It should be noted that the estimations for the acceptable velocities of the reflectors have been obtained supposing that the axial velocities of the reflectors are unknown to us. By means of the proposed technique, one can use these velocities estimation algorithm based upon the received data that is similar to the color flow principle estimations in modern ultrasound devices. In this case the acceptable velocities of the reflectors are significantly larger.

\section{CONCLuSions}

The proposed methodology for 3 -D acoustic image formation is based on the application of the 3-D fast Fourier transform. For relatively large depths, a constant width of the synthesized radiation pattern was demonstrated for a moving transducer. The technique is valid for signals with a bandwidth on the order of the center frequency.

The theory that was developed herein yields precise expressions for the spatial-temporal spectra of the receivetransmit radiation patterns of the source and receiver transducers. This theory also is useful for the development of various methods of focusing by applying various geometric schemes of scanning.

The results obtained for a spherical transducer have shown that the application of the synthetic aperture methodology for a transducer moving along only one coordinate can lead to unacceptably high side lobe levels. In the case of a transducer with a rectangular aperture, the side lobe levels do not change as the transducer moves along one coordinate or along two coordinates.

The results of the mathematical simulation and of the physical experiment coincide with the estimations obtained within the proposed theory that confirms the validity of the theory. 
The theoretical consideration of the influence of motion of the medium's scatterers was shown to influence the character of the synthesized radiation pattern. The lateral and axial motion of the scatterers modifies the radiation pattern. In the case of the lateral motion, the synthesized radiation pattern width increases or decreases depending upon the ratio of the lateral component of the scatterers' velocity and the scanning speed and upon their directions. In the case of axial motion, the radiation pattern width does not change, but its position is shifted in the lateral direction proportional to the product of the distance of the scatterer from the focus by the ratio of axial velocity and the scanning speed.

The proposed technique can be widely used in medical diagnostics in its various modifications, depending upon the field of application to achieve high-resolution 3-D images. For abdominal imaging in which the scatterers' velocity can be about $3-7 \mathrm{~cm} /$ second, it is reasonable to use a linear array that moves in a transverse direction with a cylindrical lens mounted on its surface. In this case, the influence of the scatterers' motion does not significantly change the 3-D-image resolution. For ophthalmology, dermatology, and mammography, in which the maximum depths and the examination areas are significantly smaller and the scatterers velocities are very small, it is possible to apply a spherical transducer that moves along the two orthogonal coordinates. For cardiology, further improvement of the proposed techniques seems to be possible on the basis of applying the mechanical biasing of linear phased array taking into account the velocity of scatterers by means of Doppler frequency estimation. The works in this direction are being performed within the frameworks of the research on the proposed approach to 3-D imaging.

The methodology developed in this paper can also be applied to the problems of nondestructive evaluation with the usage of higher carrier frequencies of ultrasound irradiation for better signal-to-noise ratio.

\section{REFERENCES}

[1] D. Kirbach and T. A. Whitlingham, "3D Ultrasound-the Kretztechnic Voluson ${ }^{\circledR}$ approach," Eur. J. Ultrasound, vol. 1, pp. 85-89, 1994.

[2] T. R. Nelson and D. H. Pretorius, "Three-dimensional ultrasound imaging," Ultrasound Med. Biol., vol. 24, no. 9, pp. 12431270, 1998.

[3] S. Berg, H. Torp, D. Martens, E. Steen, S. Samstad, I. Hoivik, and B. Olstad, "Dynamic three-dimensional freehand echocardiography using raw digital ultrasound data," Ultrasound Med. Biol., vol. 25, no. 5, pp. 745-753, 1999.

[4] R. Canals, G. Lamarque, and P. Chatain, "Volumetric ultrasound system for left vertical motion imaging," IEEE Trans. Ultrason., Ferroelect., Freq. Contr., vol. 46, no. 6, pp. 15271538, Nov. 1999.

[5] P. O. Corel and C. S. Kino, "A real-time synthetic aperture imaging system," Acoust. Imaging, vol. 9, pp. 341-355, 1970.

[6] C. Passmann and H. Ermert, "A $100 \mathrm{MHz}$ ultrasound imaging system for dermatologic and ophthalmologic diagnostics," IEEE Trans. Ultrason., Ferroelect., Freq. Contr., vol. 43, pp. 545-552, July 1996.

[7] C. H. Frazier and W. D. O'Brien, Jr., "Synthetic aperture techniques with a virtual source element," IEEE Trans. Ultrason., Ferroelect., Freq. Contr., vol. 45, pp. 196-207, Jan. 1998.
[8] J. T. Ylitalo and H. Ermert, "Ultrasound synthetic aperture imaging: Monostatic approach," IEEE Trans. Ultrason., Ferroelect., Freq. Contr., vol. 41, pp. 333-339, May 1994.

[9] Z. M. Benenson, N. S. Kulberg, and T. T. Kasumov, "A new approach to obtain non-diffraction beam with near-field resolution on linear and convex arrays," Acoust. Imaging, vol. 23, pp. 303-308, 1997.

[10] J. A. Jensen, "A model for the propagation and scattering of ultrasound in tissue," J. Acoust. Soc. Amer., vol. 89, pp. 188191, 1991.

[11] C. R. Hill, Physical Principles of Medical Ultrasound. Chichester, UK: Ellis Horwood, 1986.

[12] C. S. Kino, Acoustic Waves: Devices, Imaging and Analog Signal Processing. Englewood Cliffs, NJ: Prentice-Hall, 1987.

[13] J.-Y. Lu, M. Fatemi, and J. F. Greenleaf, "Pulse-echo imaging with X-waves," Acoust. Imaging, vol. 22, pp. 191-196, 1996.

[14] J.-Y. Lu, "2D and 3D high frame imaging with limited diffraction beams," IEEE Trans. Ultrason., Ferroelect., Freq. Contr., vol. 44, no. 4, pp. 839-856, July 1997.

[15] S. W. Smith, H. C. Pavy, Jr., and O. T. von Ramm, "Highspeed ultrasound volumetric imaging system. Part I: Transducer design and beem steering," IEEE Trans. Ultrason., Ferroelect., Freq. Contr., vol. 38, pp. 100-108, Mar. 1991.

[16] O. T. von Ramm, S. W. Smith, and H. C. Revy, Jr., "Highspeed ultrasound volumetric imaging system. Part II: Parallel processing and image display," IEEE Trans. Ultrason., Ferroelect., Freq. Contr., vol. 38, pp. 109-115, Mar. 1991.

[17] R. E. Davidsen and S. W. Smith, "Sparse geometry for twodimensional array transducers in volumetric imaging," in Proc. IEEE Ultrason. Symp., 1993, pp. 1091-1094.

[18] J. W. Goodman, Introduction to Fourier Optics. New York: McGraw Hill, 1968.

[19] C. Passmann, H. Ermert, T. Auer, K. Kaspar, S. el-Gammal, and P. Altmeyer, "In vivo ultrasound biomicroscopy," in Proc. IEEE Ultrason. Symp., 1993, pp. 1015-1018.

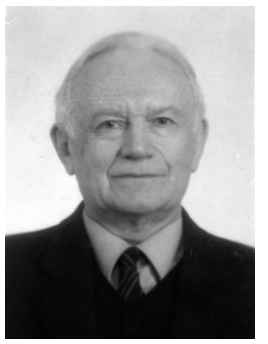

Zalman M. Benenson was born in Borisov, USSR, on March, 4, 1922. He graduated from Moscow State University in 1942 and Artillery Academy in 1944. He received the Ph.D. and Doctor of Science degrees in 1947 and 1956, respectively. From 1945-1958, he worked in the field of radars and control devices research and design for anti-aircraft defense. From 1958-1970, he worked in research and design of radars information processing and automated control systems for anti-aircraft defense of the USSR. From 1971-1984, he worked in the field of computer aided design and signal processing systems investigations for radars and lasers. Since 1985 he has been head of the department "Theory and Algorithms of Signal Processing" in the Scientific Council on Cybernetics of the Russian Academy of Science.

His scientific interests are radar signals processing, ultrasound and laser signals processing, mathematical simulation of coherent signal propagation in various media, algorithmic image analysis and synthesis in medical ultrasound and medical radiology. He is the author of 4 books and 115 papers He is a Laureate of the USSR State Prize, a member of International Academy of Informatisation, and Professor of the Moscow Institute of Physics and Technology.

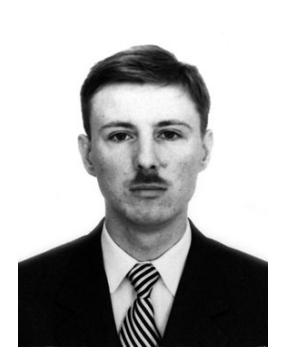

Alexey B. Elizarov was born in Moscow, Russia, on November 15, 1976. He graduated with honors from the Moscow Institute of Physics and Technology (Moscow) in 1999. Since 1997 he has worked in the Scientific Council on Cybernetics of the Russian Academy of Science (SCC RAS). From 19971999 he worked part-time as engineer in SCC RAS in the field of digital ultrasound signals and X-rays images processing for the purposes of medical diagnostics. Since 1999 he has been a postgraduate student and researcher of SCC RAS. 
His area of expertise is digital signal processing and computer simulation of ultrasound signals for the purposes of medical diagnostics.

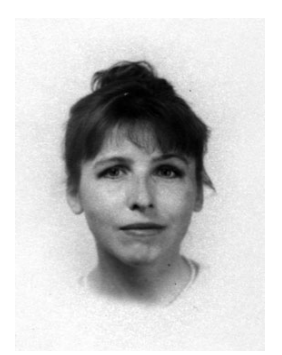

Tatiana V. Yakovleva was born in Magnitogorsk, Russia on July 5, 1956. She graduated with honors from the Moscow Engineering Physics Institute (Moscow) in 1979. She defended her Ph.D. thesis in Physics and Mathematics in the Physics Institute of the Soviet Academy of Science, Moscow, in 1982. The topic of the thesis was theoretical study of nonlinear optical effects in volume holography and wave front conjugation. Since 1986 she has worked in the Scientific Council on Cybernetics of the Russian Academy of Science. Her area of expertise until 1990 was theoretical investigations in the field of non-linear optics, induced scattering of light, study of frequency shift caused by the process of light propagation. Since 1990 her area of expertise has been ultrasound propagation through biological medium, ultrasound imaging with high resolution and nondiffractive pulse propagation From 1993-1994 she conducted research work in London, Department of Medical Physics, St. George's Hospital as a British Royal Society postdoctoral fellow (she was awarded a 12-month British Royal Society Postdoctoral Fellowship). The field of the research was the investigation in the field of ultrasound scattering by inhomogeneous biological structure (liver) for medical ultrasound diagnostics.

Currently Tatiana V. Yakovleva works as a senior scientist of the Scientific Council on Cybernetics of the Russian Academy of Science in the field of theoretical elaboration and computer simulation of ultrasound visualization process and digital signal processing for the purposes of medical diagnostics.

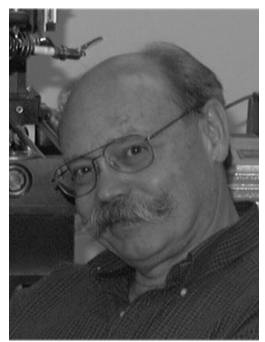

William D. O'Brien, Jr. (S'64-M'70SM'79-F'89) received the B.S., M.S., and Ph.D. degrees in 1966, 1968, and 1970, from the University of Illinois, Urbana-Champaign.

From 1971 to 1975 he worked with the Bureau of Radiological Health (currently the Center for Devices and Radiological Health) of the U.S. Food and Drug Administration. Since 1975, he has been at the University of Illinois, where he is Professor of Electrical and Computer Engineering and of Bioengineering, College of Engineering; Professor of Bioengineering, College of Medicine; Professor of Nutritional Sciences, College of Agricultural, Consumer and Environmental Sciences; a Research Professor in the Beckman Institute for Advanced Science and Technology; and a Research Professor in the Coordinated Science Laboratory. He is the Director of the Bioacoustics Research Laboratory. His research interests involve the many areas of ultrasoundtissue interaction, including spectroscopy, risk assessment, biological effects, tissue characterization, dosimetry, blood-flow measurements, acoustic microscopy and imaging for which he has published 246 papers.

Dr. O'Brien is a Fellow of the Institute of Electrical and Electronics Engineers, the Acoustical Society of America and the American Institute of Ultrasound in Medicine, and a Founding Fellow of the American Institute of Medical and Biological Engineering. He was recipient of the IEEE Centennial Medal (1984), the AIUM Presidential Recognition Awards (1985 and 1992), the AIUM/WFUMB Pioneer Award (1988), the IEEE Outstanding Student Branch Counselor Award for Region 4 (1989), the AIUM Joseph H. Holmes Basic Science Pioneer Award (1993), and the IEEE Ultrasonics, Ferroelectrics, and Frequency Control Society Distinguished Lecturer (1997-1998). He received the IEEE Ultrasonics, Ferroelectrics, and Frequency Control Society's Achievement Award for 1998, and the IEEE Millennium Medal in 2000. He has served as Co-Chair of the 1981 and 2001 IEEE Ultrasonic Symposia, and General Chair of the 1988 IEEE Ultrasonics Symposium. He is Co-Chair of the 2003 IEEE Ultrasonics Symposium. He has been Secretary-Treasurer (1972-1980), Vice President (1981), and President (1982-1983) of the IEEE Sonics and Ultrasonics Group (currently the IEEE Ultrasonics, Ferroelectrics, and Frequency Control Society), and Editor-inChief of the IEEE Transactions on Ultrasonics, Ferroelectrics, and Frequency Control (1984-2001). He has been Treasurer (1982-1985), President-Elect (1986-1988) and President (1988-1991) of the American Institute of Ultrasound in Medicine. He has served on the Board of Directors (1988-1993) of the American Registry of Diagnostic Medical Sonographers, and has been Treasurer (1991-1994) of the World Federation for Ultrasound in Medicine and Biology. 